\title{
Simulation of air invasion in immersed granular beds with an unresolved FEM-DEM model
}

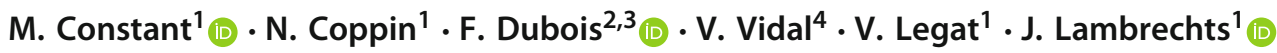

\begin{abstract}
This paper is devoted to an unresolved model for the simulation of air invasion in immersed granular flows without interface reconstruction between the liquid and the gas. Experiments of air invading a granular bed immersed in ethanol were achieved in a Hele-Shaw cell to observe the gas invasion paths and to calibrate the numerical multiscale model. The grains movements are computed at a fine scale using the non-smooth contact dynamics method, a time-stepping method considering impenetrable grains. The fluid flow is modelled by equations averaged using the volume fraction of fluid and computed at a coarse scale with the finite element method. A phase indicator function is used to dissociate the gas and the liquid constituting the fluid and to compute the density and viscosity of the fluid at each position. It is moved using a convection equation at each time step. The fluid, solid and phase indicator function computations are validated on simple cases before being used to reproduce experiments of air invasion in immersed granular flows. The experiments are supported by simulations in two dimensions to refine the study and the understanding of the invasion dynamics at short times.
\end{abstract}

Keywords Finite element $\cdot$ Discrete element $\cdot$ Fluidized bed $\cdot$ Multiphase flows $\cdot$ MigFlow

\section{Introduction}

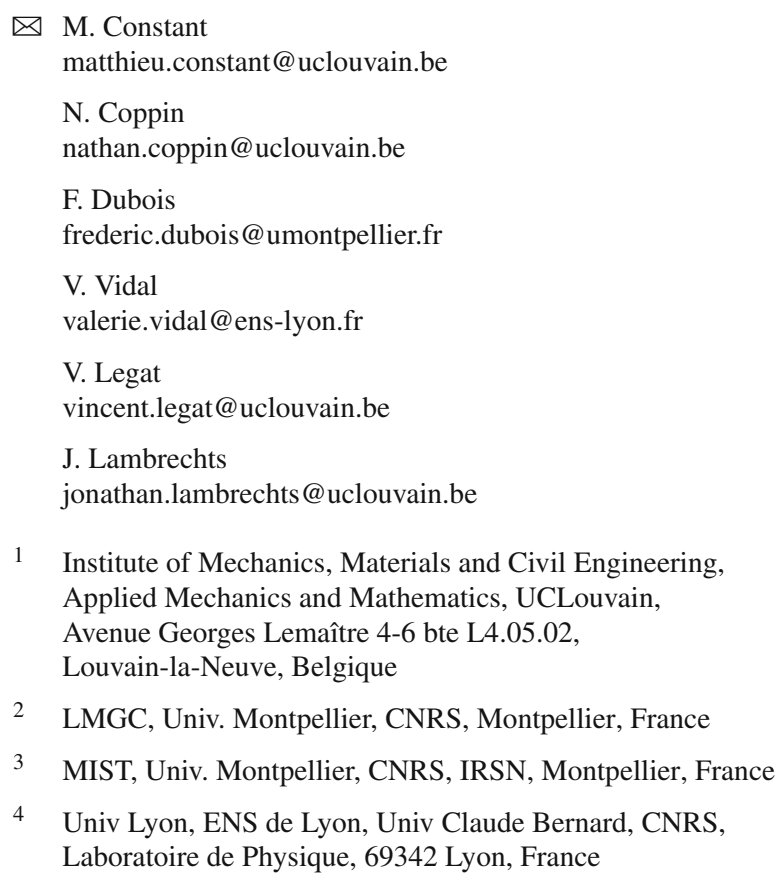

This research was funded by the F.R.S.-FNRS through a FRIA Grant.
Flows of grains surrounded by fluid also called immersed granular flows are a widespread research and industrial topic. Features of immersed granular flows are as various as the processes in which they appear. The presence of discrete solid grains complicates the flow by creating heterogeneous mixtures ranging from pure liquid to porous medium. The impact of the highly inhomogeneous nature of fluid-grains mixtures on the flow regimes is the source of the lack of knowledge about immersed granular flows [64]. The present study was carried out in the framework of the MigFlow ${ }^{1}$ project with the aim of developing and validating a free opensource software to compute immersed granular flows.

Numerical methods are useful tools to study and to understand how small-scale behaviours of immersed granular flows affect large-scale properties of their dynamics. Immersed granular flows are used in many different fields such as chemical engineering, agro-food industry, civil engineering and geology. Several mathematical models have been suggested to compute their motion depending on the application scale $[17,60]$. Either the movements of the grains can

\footnotetext{
1 www.migflow.be.
} 
be modelled explicitly at their scale using a discrete model or their flow can be represented at a larger scale using a continuous model. The same goes for the fluid flow that can be computed at a small scale between the grains or averaged at a coarser scale using a mixture representation of the flow. Large-scale computations make use of continuum models. Balance equations are used to compute the momentum transfer between the two continuous phases like in the so-called two-fluid model [22]. Continuum models are computationally convenient, but they require constitutive or closure equations [70] that strongly restrain their use to specific phenomenological assumptions. Small-scale computations make use of discrete models. A wide variety of state equations and contact solvers for the grains dynamics have been coupled to an equally high number of fluid description models [71] in order to create different models for immersed granular flows characterized by the fluid computation scale.

Considering grains as Lagrangian particles requires to compute the resultant force causing their motion at all times. However, moving grains in multibody systems cannot be achieved without taking the neighbouring grains into account. The presence of neighbours constrains the displacement of the grains. To simulate a grain movement in a porous medium requires to detect collisions in order to prevent any interpenetrations. A first approach to consider collisions is to determine the time at which collisions occur and compute them sequentially. In densely grain charged flows, such event-driven methods [38] are numerically impracticable because the time interval between collisions is too small. When considering dense granular media, time-stepping methods are used to solve all the contacts happening during a given time step. Two possible grain representations are mainly used to face this difficulty. It is possible to move the grains allowing small interpenetrations by adding a repulsive force between overlapped grains like in the smooth discrete-element method (DEM) [14]. It creates an explicit collisions solver that is numerically convenient because the spring model used for the repulsive force between overlapped grains is based on the magnitude of the previous time step. It is necessary to introduce damping in such models to smooth persistent oscillations in fully deposed granular beds [14]. On the contrary, it is possible to use non-smooth DEM [30]. In this case, grains interpenetrations and deformations are prevented by modifying the velocities of the grains to find at each time step a set of velocities producing a stationary state of the grains without interpenetrations.

As far as the fluid is concerned, different models can also be used [71]. Fully resolved methods make use of a spatial discretization of the fluid domain that is finer than the grains size $[27,41,69]$. Those very accurate methods require fewer phenomenological laws and are often used to calibrate methods on coarser grids. These methods have an important numerical cost due to the high number of unknowns.
In 1992, Tsuji et al. [59] laid the mathematical foundation of a Lagrangian-Eulerian representation based on a coarser representation of the fluid than the grain scale. This kind of hybrid unresolved approach is convenient to solve immersed granular flows presenting a large range of volume fractions $[10,11,70]$. They are based on a Lagrangian representation of the grains at their scale while the fluid flow is computed from Navier-Stokes equations averaged on a control volume greater than the grains scale. This Eulerian representation of the fluid speeds the numerical resolution of the fluid equations up, while the Lagrangian representation of the grains gives insight in the microscale effects due to the grains configuration. The difficulty of this kind of model is to choose an appropriate interaction force relationship to couple the grains motion with the fluid flow. The momentum exchange cannot be directly computed at the grain scale by integrating the force at the solid-fluid interface because the fluid is not represented at this scale [32]. Different interaction force relationships have been proposed, characterised by the formulation of the drag force and the repartition of the pressure gradient on both phases [64].

This class of Lagrangian-Eulerian models has been intensively used to understand the variables of interest and describe the gas dynamics in fluidized beds $[32,37,68,72]$. These studies were limited to the description of flows mixing grains with a single fluid (see [7] for simulations of particleladen flows using the finite element method coupled to the discrete-element method). More recent papers deal with the problem of three-phase flows using techniques to capture the interface between gas and liquid $[39,43,50,61]$.

Recently, a growing attention has been paid to the fluidization of immersed granular beds because of their use in processes like grains drying, sorting or mixing [19] but also in environmental applications like air sparging in which air is injected into the ground to fracture, degrade and carry away some contaminants during its ascend [48] and in geological phenomena like mud volcanoes or hydrothermal vents [51]. The fluidized area is challenging to observe in three-dimensional granular beds so that state-of-the-art non-intrusive imaging techniques are required [34]. That is why quasi-two-dimensional devices like Hele-Shaw cells are favoured in research dealing with the fluidized area morphology [63]. Experiments of gas injection in granular beds immersed in water give the opportunity to classify the different fluidized area morphology with respect to the inflow rate and the effective gravity [62]. However, the transitions between the different regimes such as percolation and fracture are not so clear. There exists a range of inflow rates and effective gravity for which it is not possible to predict the fluidized chimney behaviour due to the importance of the medium heterogeneities and local grains distribution on the invasion dynamics [62]. Characterizing experimentally or numerically the morphology of a fluidized area like the ocean 
floor can give insight into geological observations. Until now, only simplified and empirical models have been used to simulate the gas injection process in immersed granular beds. For example, Varas et al. [63] use a network representation of the granular matrix in which the nodes are the grains interstitial gap and the edges between those gaps are weighted by capillary and hydrostatic pressures [63].

This paper deals with the implementation and the application of an unresolved CFD-DEM model to simulate the gas injection in granular beds immersed in a fluid without interface reconstruction. Comparisons with experiments are provided to validate the model accuracy. The objective is to validate the model implemented in the open-source software MigFlow. It provides a new tool to investigate the formation of the fluidized area and the constraints propagation inside the granular pile compared to empirical models previously used to describe the system. New numerical and experimental data of gas invasion in an immersed granular medium are reported. The laboratory set-up consists of a quasi-two-dimensional device (Hele-Shaw cell); two-dimensional simulations are used to compute the process in order to determine the features reproducible in spite of the loss of a dimension. In Sect. 2, the equations of the model are developed. The choice of the finite element method (FEM) used for the spatial discretization of the fluid equations will be discussed. A particular attention will be paid to the stability of the method. In order to deal with the explicit coupling and the stiff interaction forces between the phases, a new predictor-corrector scheme is developed in Sect. 3. Textbook cases are reproduced in order to assess the accuracy of the unresolved model and the relevance of the numerical scheme. Finally, Sect. 4 shows the two-dimensional simulations of the air invasion in immersed granular beds and the comparisons with experiments achieved in a Hele-Shaw cell.

\section{Unresolved FEM-DEM model}

Multiscale models are frequently used to compute immersed granular flows $[10,11,59,70]$. Grains are solved at their scale and considered as rigid Lagrangian particles, while the fluid is computed at a coarser scale by smoothing spatial discontinuities coming from the grains to obtain a continuous fluid representation. The first part of this section is devoted to the fluid equations of the multiscale model and their spatial discretization. The objective is to simulate the interactions between liquid, gas and solid phases. The model is extended in the second part to take into account a fluid with two constituents. Fluid refers to a continuous mixture of the liquid and gas constituents so that the fluid extensive properties like the density and the viscosity vary in space and time. The surface tension force is written in the third part along with a validation case to demonstrate the accuracy of the numeri- cal model. The fourth part concerns the grains representation and the computation of the contacts between the rigid bodies. Finally, the fluid-grains interaction force is discussed in the fifth part and illustrated by computing the settling velocity of a single grain.

\subsection{Fluid-phase model}

Fluid properties are averaged over a control volume larger than the grains. Following the averaging process suggested by Anderson and Jackson [1], the integral of a fluid property is substituted by the mean value of this property multiplied by the porosity, i.e. the fluid volume fraction $\phi$. The expressions of the time and spatial derivatives of the fluid local mean values give the averaged Navier-Stokes equations of the fluid phase:

$$
\begin{aligned}
& \frac{\partial \phi}{\partial t}+\nabla \cdot \boldsymbol{u}=0 \\
& \frac{\partial \rho \boldsymbol{u}}{\partial t}+\nabla \cdot \frac{\rho \boldsymbol{u} \boldsymbol{u}}{\phi}=\nabla \cdot[2 \mu \phi \boldsymbol{d}-p \boldsymbol{I}]+\boldsymbol{f}+\boldsymbol{T}+\phi \rho \boldsymbol{g}
\end{aligned}
$$

where $\rho$ is the density, $\mu$ is the dynamic viscosity, $\boldsymbol{u}$ is the fluid velocity multiplied by $\phi, p$ is the pressure, $\boldsymbol{I}$ is the identity tensor, $\boldsymbol{f}$ is the fluid-grain interaction force, $\boldsymbol{T}$ is the surface tension force, $\boldsymbol{g}$ is the gravity and $\boldsymbol{d}$ is the symmetric gradient of $\boldsymbol{u} / \phi$.

The spatial discretization of those equations is achieved on unstructured grids using the finite element method. Let us define $\boldsymbol{U}^{h}$ and $\mathcal{P}^{h}$ some suitable trial solution spaces for the velocity and the pressure fields along with $\hat{\boldsymbol{U}}^{h}$ and $\hat{\mathcal{P}}^{h}$ some suitable test function spaces for the velocity and pressure fields on a triangulation containing $N$ elements and $N_{n}$ nodes. Velocity and pressure are written:

$\boldsymbol{u} \simeq \boldsymbol{u}^{h}=\sum_{i}^{N_{n}} \boldsymbol{U}_{i} \tau_{i}, \quad \boldsymbol{u}^{h} \in \boldsymbol{U}^{h}$

$p \simeq p^{h}=\sum_{i}^{N_{n}} P_{i} \tau_{i}, \quad p^{h} \in \mathcal{P}^{h}$,

where $\tau_{i}$ are linear interpolation functions, while $\boldsymbol{U}_{i}$ and $P_{i}$ are the nodal values of the velocity and pressure fields. At each time, the nodal values of the porosity $\Phi_{i}$ are computed from the grains position as

$\Phi_{i}=1-\frac{1}{\int_{\Omega} \tau_{i}(\mathbf{x}) \mathrm{d} \mathbf{x}} \sum_{s \in \mathcal{S}} V_{s} \tau_{i}\left(\mathbf{x}_{s}\right), \quad \forall i \in N_{n}$,

where $\mathcal{S}$ is the set of grains and $V_{S}$ (resp. $\mathbf{x}_{S}$ ) is the volume (resp. position) of the grain $s$. As a result, the only unknowns are the fluid velocity and pressure. From this equation, it 
also appears that the chosen averaging scale of the averaged Navier-Stokes equations is linked to the fluid discretization scale.

Linear equal-order interpolation functions (P1-P1) are used in order to preserve the numerical efficiency inherent to the use of a multiscale model. It also provides low order interpolations of the fluid fields that are suitable for the multiscale representation of the fluid-grains interaction. This combination of interpolation functions induces numerical instabilities in the form of spurious modes in the pressure field [57]. The use of P1-P1 elements to solve Navier-Stokes equations does not respect the Brezzi-Babuska condition $[2,4]$ and provides a singular discrete matrix. Filling the deficient rank of this matrix can be done by adding a stabilizing term in the continuity equation referred to as the pressurestabilizing/Petrov-Galerkin (PSPG) term [28,57].

Another difficulty comes from the non-positive definite matrix associated with the convective terms that breaks the best approximation property of the finite element method and corrupts the solution by creating oscillations in the velocity field [5]. It is possible to limit the numerical adverse effects of the convective terms by adding just enough numerical diffusion in the flow direction. This formulation is usually referred to as the streamline upwind/Petrov-Galerkin (SUPG) formulation. The addition of the SUPG term in the momentum equations is equivalent to modify the weighting functions of the Petrov-Galerkin formulation using the velocity field and to use these modified weighting functions for all terms in the momentum equations giving a consistent formulation [5].

The drawback of the addition of stabilizing terms is that it could destroy the accuracy if they become dominant even if the SUPG and PSPG formulations are based on the derivative of the following continuity and momentum equations residuals:

$$
\begin{aligned}
R_{p}\left(\boldsymbol{u}^{h}\right) & =\frac{\partial \phi}{\partial t}+\nabla \cdot \boldsymbol{u} \\
\boldsymbol{R}_{\boldsymbol{u}}\left(\boldsymbol{u}^{h}, p^{h}\right) & =\frac{\partial \rho \boldsymbol{u}^{h}}{\partial t}+\nabla \cdot \frac{\rho \boldsymbol{u}^{h} \boldsymbol{u}^{h}}{\phi} \\
& +\nabla p^{h}-\boldsymbol{f}^{h}-\boldsymbol{T}^{h}-\phi \rho \boldsymbol{g}
\end{aligned}
$$

and provide convergent methods. The viscous term has been removed from the momentum residual because its derivative is zero for linear interpolation functions. That is why the choice of the stabilization parameters is a problem that has been intensively treated in the past literature [36,49,56-58]. Moreover, the PSPG term added in the continuity equation may have devastating consequences if the loss of the incompressibility flow property is not limited. The incompressibility is enforced by introducing in the momentum equation a penalty term based on a least-square estimator of the continuity equation residual [58]. This term is referred to as the least square on incompressibility constraint (LSIC) term.

The stabilized finite element formulation of the averaged Navier-Stokes equations is to find $\left(\boldsymbol{u}^{h}, p^{h}\right) \in\left(\boldsymbol{U}^{h} \times \mathcal{P}^{h}\right)$ such that $\forall\left(\hat{\boldsymbol{u}}^{h}, \hat{p}^{h}\right) \in\left(\hat{\boldsymbol{U}}^{h} \times \hat{\mathcal{P}}^{h}\right)$ :

$$
\begin{aligned}
& <\frac{\partial \phi}{\partial t}, \hat{p}^{h}>-<\boldsymbol{u}^{h}, \nabla \hat{p}^{h}>+\ll \boldsymbol{u}^{h}, \hat{p}^{h} \boldsymbol{n} \gg \\
& =-\overbrace{\sum_{e=1}^{N} \xi_{p}<\boldsymbol{R}\left(\boldsymbol{u}^{h}\right), \nabla \hat{p}^{h}>_{e}}^{\text {PSPG }} \\
& <\frac{\partial \rho \boldsymbol{u}^{h}}{\partial t}, \hat{\boldsymbol{u}}^{h}>+<\nabla \cdot\left(\frac{\rho \boldsymbol{u}^{h} \boldsymbol{u}^{h}}{\phi}+p^{h} \boldsymbol{I}\right), \hat{\boldsymbol{u}}^{h}> \\
& =<2 \mu \phi \boldsymbol{d}^{h}, \nabla \cdot \hat{\boldsymbol{u}}^{h}>-\ll 2 \mu \phi \boldsymbol{d}^{h}, \hat{\boldsymbol{u}}^{h} \cdot \boldsymbol{n} \gg \\
& +<\boldsymbol{f}^{h}+\boldsymbol{T}^{h}+\phi \rho \boldsymbol{g}, \hat{\boldsymbol{u}}^{h}> \\
& -\underbrace{\sum_{e=1}^{N} \xi_{s}<\boldsymbol{R}\left(\boldsymbol{u}^{h}\right), \boldsymbol{u}^{h} \cdot \nabla \hat{\boldsymbol{u}}^{h}>_{e}}_{\text {SUPG }} \\
& -\underbrace{\sum_{e=1}^{N} \xi_{c}<R_{p}\left(\boldsymbol{u}^{h}\right), \nabla \cdot \hat{\boldsymbol{u}}^{h}>_{e}}_{\text {LSIC }},
\end{aligned}
$$

where the notations $\langle\cdot, \cdot\rangle$ and $\ll \cdot, \cdot \gg$ are used for the inner products on the domain $\Omega$ and its boundary $\partial \Omega$, respectively. The subscript $e$ in $\langle\cdot, \cdot\rangle_{e}$ denotes the restriction of the inner product to the interior of the element $e$.

The stabilization parameters are obtained from element matrices and vectors [58]:

$$
\begin{aligned}
& \xi_{p}=\xi_{s}=\left[\left(\frac{2}{\Delta t}\right)^{2}+\left(\frac{\left\|\boldsymbol{u}^{h}\right\|}{\bar{h}}\right)^{2}+\left(\frac{4 v}{\bar{h}^{2}}\right)^{2}\right]^{-\frac{1}{2}} \\
& \xi_{c}=h\left\|\boldsymbol{u}^{h}\right\| \min \left(\frac{h \rho\left\|\boldsymbol{u}^{h}\right\|}{6 \mu}, \frac{1}{2}\right) .
\end{aligned}
$$

\subsection{One fluid with two immiscible phases}

The previous parts describe the evolution of the bulk velocity and pressure of the fluid for given density and viscosity fields. In this part, we consider a fluid with two immiscible phases so that the fluid extensive properties vary spatially. An equation is needed to describe the evolution of these fields and the interface motion between the two immiscible phases. The treatment of the discontinuities between the two fluid phases is similar to the treatment of the grains.

A phase indicator function $\alpha$ is chosen as equal to one in the first phase and zero in the other one. This is a sharp function whose steps defined the boundaries between the two constituents. The problem consists in capturing these bound- 
aries in a discrete way and solving their evolution in time along with the balance equations between the phases. Taking an Eulerian point of view, it is quite difficult to follow accurately a moving boundary on a fixed mesh because the advective fluxes carrying the interface are averaged over a control volume. This averaging process blurs the interfaces so that it is appropriate to work with the average value $a$ of the phase indicator function that represents the volume fraction of the first constituent $[24,26,46]$. The surface between the constituents is then defined by cells with a value of $a$ that is between zero and one. This model is sometimes referred to as the one-fluid formulation of a two-fluid-phase flow [44]. The evolution of this volume fraction function can be understood by considering the Lagrangian representation of the problem. In this case, each mechanical point under consideration moves with the flow so that if a point belongs to the constituent $i$ at the beginning of the computation, this point belongs to the constituent $i$ until the end of the computation. In the Eulerian representation, the evolution of the volume fraction in each cell is given by:

$$
\frac{\partial a \phi}{\partial t}+\nabla \cdot(a \boldsymbol{u})=0
$$

The presence of a second continuous phase is taken into account in the fluid Eqs. (1) and (2) by weighting the constituents extensive properties by the volume fraction. Even if the linear interpolation of the density can be directly deduced from the sum of the mass conservation law of each fluid, the choice between the interpolation of the kinematic viscosity or the dynamic viscosity is still questionable in the vicinity of the interface [44]. For the purpose of this work, we chose:

$\rho=a \rho_{1}+(1-a) \rho_{2}$,

$\mu=\left(a \frac{\mu_{1}}{\rho_{1}}+(1-a) \frac{\mu_{2}}{\rho_{2}}\right) \rho$.

We previously discussed the stabilization of the classical finite element formulation for equations with dominant convection terms. Equation (12) is subject to the same difficulties. A similar stabilization formulation could have been chosen to solve this equation, but it would have come with extra diffusion of the volume fraction. To solve the NavierStokes equations, this numerical diffusion is insignificant compared to the diffusion coming from the viscous terms. However, the coarse-scale representation of the fluid already blurs the interface, and an additional diffusion due to the numerical scheme is the last thing we want in the computation of the volume fraction. The discontinuous Galerkin method has been chosen for its efficiency to represent convection without extra numerical diffusion.

\subsection{Surface tension force}

The computation of the surface force applied on the interface comes along with the identification of the interface. In the context of this work, the interface is located in areas with high variations of the phase indicator function in a frontcapturing way. It is not possible to apply the surface tension force on the discrete interface so that we are interested in applying a volume force on the fluid. This force is significant where the phase indicator function gradient is high and cancels where the indicator function is constant. This is the base of the continuum surface force model (CSF) developed by Brackbill et al. [3]. It is based on a volume reformulation $\boldsymbol{T}$ of the surface tension force in which the curvature interface $\kappa$ and the normal to the interface $\boldsymbol{n}$ are computed using the phase indicator function:

$\boldsymbol{T}=\sigma \overbrace{\nabla \cdot \underbrace{\left(-\frac{\nabla a}{\|\nabla a\|}\right)}_{n}}^{\kappa} \nabla a$,

where $\sigma$ is the surface tension coefficient. The drawback of this method is the creation of spurious currents [35] near the interface. Due to the mesoscale discretization of the fluid, the representation of a thin interface is underresolved which results in an imbalance in the advection, viscous and capillary pressure tensors in its neighbourhood. Various methods have been proposed to reduce spurious currents such as a smoothing of the phase indicator function [35], a consistent discretization of the pressure-gradient based on an accurate representation of the interface [42] or an energy conserving discretization [29]. In the framework of this study, the spurious currents take a long time to become significant. If this time is longer than the experiment duration or the relevant time for the observations, it is not mandatory to use such stabilization strategies. A similar simulation to the computation of the pressure difference across a bubble interface presented below has been achieved using the same numerical and physical parameters than those used to compute the air invasion of immersed granular beds. It reveals that it takes much more time for the spurious currents to become dominant than the time taken by a single bubble to rise to the top of the cell (i.e. to go out of the computational domain) during the air invasion process.

\subsubsection{Pressure difference across a bubble interface}

Let us consider a textbook case to validate our computation. The surface tension force creates a pressure difference across the interface separating a liquid and a gas. For the case of an infinite cylindrical rod, this pressure difference can be computed analytically. Without gravity and with the liquid 

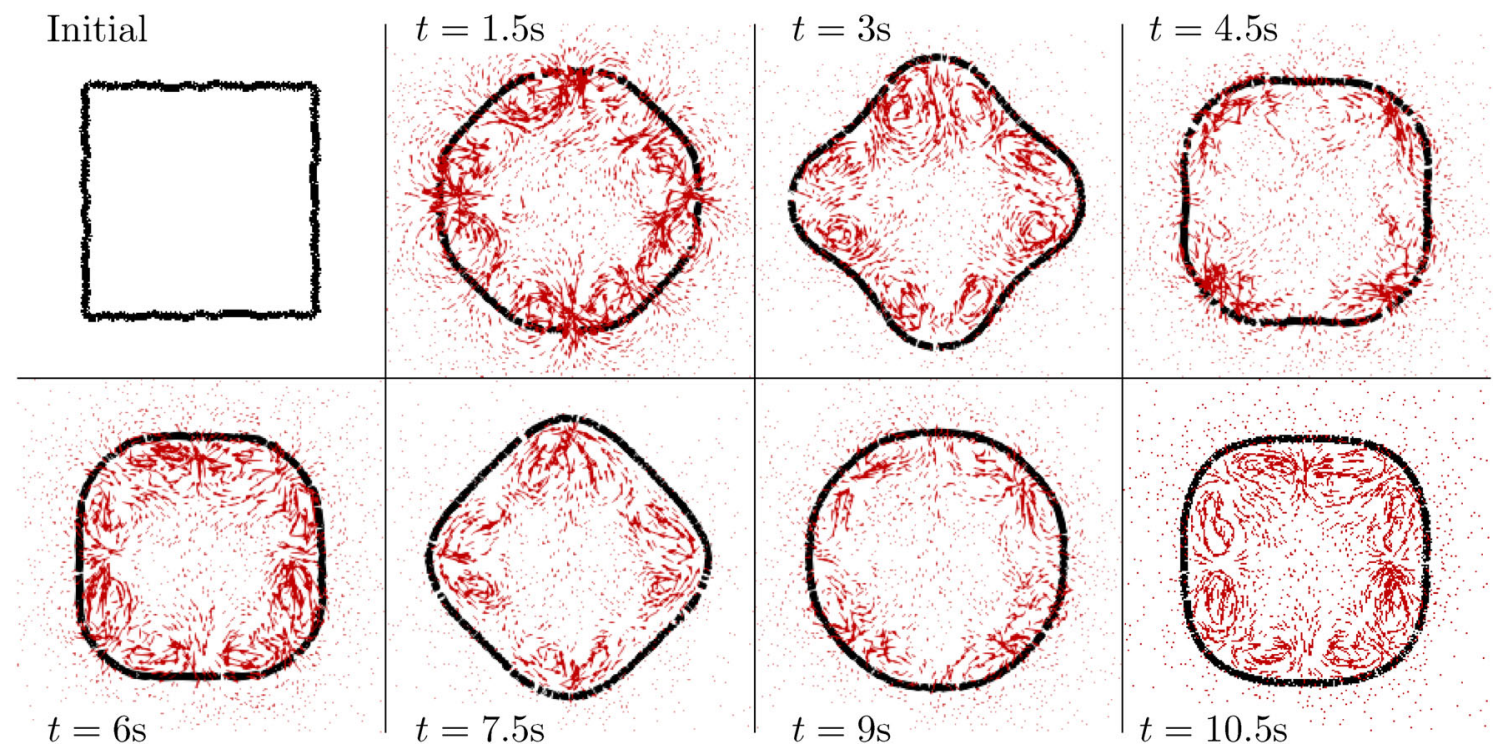

Fig. 1 Time evolution of an air drop with initial square shape surrounded by ethanol in the absence of gravity. A constant time step of $1.5 \mathrm{~s}$ is taken between successive snapshots. The interface is represented

by the black line on the pictures where the phase indicator function is equal to 0.5 . Red arrows represent the fluid velocity field. (Color figure online)

at rest, the solution of the momentum equation leads to the well-known Laplace's relationship:

$p_{\text {in }}-p_{\text {out }}=\frac{\sigma}{R}$,

where $p_{\text {in }}$ and $p_{\text {out }}$ are, respectively, the pressure inside and outside the cylinder and $R$ is the cylinder radius.

Let us consider an air drop $\left(\rho_{\mathrm{g}}=1.117 \mathrm{~kg} / \mathrm{m}^{3}\right.$ and $\mu_{\mathrm{g}}=1.75 \times 10^{-5} \mathrm{~Pa} \mathrm{~s}$ ) with an initial square shape in ethanol ( $\rho=785 \mathrm{~kg} / \mathrm{m}^{3}$ and $\mu=1.2 \times 10^{-3} \mathrm{~Pa} \mathrm{~s}$ ). Without gravity, the surface tension force tends to smooth the corners until the drop has a circular shape. The shape of the bubble oscillates around the circle due to the strong curvature of the interface, i.e. the strong surface tension force at the corners. Viscous effects damp the oscillations forcing the drop to tend towards an equilibrium circular state. The time evolution of the square drop is presented in Fig. 1, while Fig. 2 shows the final state of the drop. As stated above, it can be seen that after a long time of simulation, spurious currents create oscillations in the pressure field, but for the application we will be interested in later air bubbles reach the top surface and go out the computational domain faster than the time needed for the oscillations to become significant.

Starting with a square air drop of side $L=\sqrt{\pi} / 10 \mathrm{~m}$, the radius of the equilibrium circular drop obtained is $R=0.1 \mathrm{~m}$. Assuming that the air-ethanol surface tension is $\sigma=22.39 \times$ $10^{-3} \mathrm{~N} / \mathrm{m}$ and the pressure in the surrounding ethanol far from the drop is zero, the pressure in the circular drop should be $p_{\text {in }}=0.2239 \mathrm{~Pa}$ according to the Laplace's law (16) validating the numerical result presented in Fig. 2. This result has been obtained using an element size 20 times smaller than the drop radius. In applications dealing with grains, one should remember that elements must be larger than the grains. The resolution we could obtain for air bubbles invading an immersed granular bed therefore depends on the relative size of the grains and the bubbles.

\subsection{Solid-phase model}

Contacts are computed with the non-smooth contact dynamics (NSCD) method [30]. The starting point of the method is the second Newton's law of motion stating the evolution of each spherical grain $s$ of the set of grains $\mathcal{S}$ :

$m_{s} \frac{\mathrm{d} \boldsymbol{u}_{s}}{\mathrm{~d} t}=m_{s} \boldsymbol{g}+\boldsymbol{F}_{s}+\sum_{j \in\{\mathcal{S} \backslash s\}} \boldsymbol{R}_{s j}^{c}$,

where $m_{s}$ and $\boldsymbol{u}_{s}$ are, respectively, the mass and the velocity of the grain $s$, while $\boldsymbol{F}_{s}$ is the force applied by the fluid on the grain and $\boldsymbol{R}_{s j}$ is the contact reaction force exerted by the grain $j$ on the grain $s$. For the sake of concision, the contact reaction forces exerted by the boundaries on the grains are not presented in the above formula because they are treated like the grain-grain reaction forces.

The NSCD method consists in finding the reaction forces using an inelastic contact law before moving the grains to 


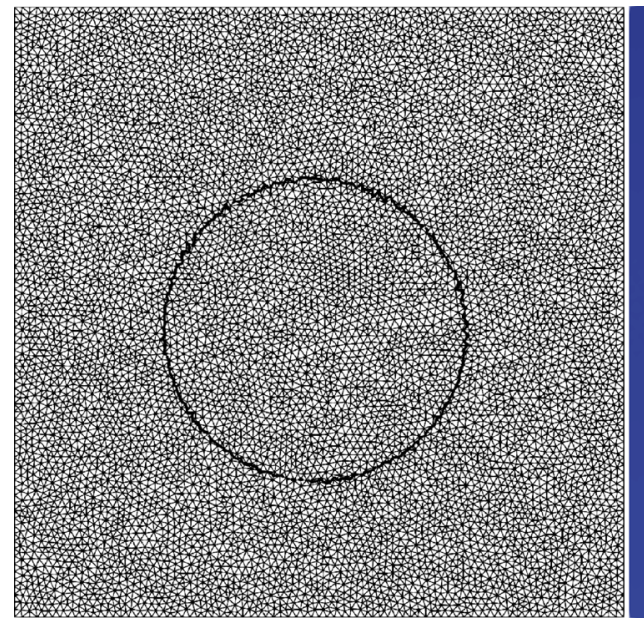

Fig. 2 State of the air drop with an initial square shape surrounded by ethanol at $t=40 \mathrm{~s}$. Left figure shows the mesh used for the computation, and right figure shows the pressure field. The interface is the black line located where the phase indicator function is equal to 0.5 . Oscillations in the pressure field can be seen near the interface where spurious currents are located obtain a set of velocities so that when the resultant forces of the external forces and reaction forces are applied to move the grains, there is no interpenetration. This method is emphasized in this work rather than the classical springdashpot model of the smooth DEM [14] because it allows to use larger time steps. It reduces the number of sub-time steps used to solve the contacts at each fluid step. Moreover, the non-smooth model better matches the hypothesis of an incompressible mixture.

The computational method we used to solve the contact reaction forces and move the grains is the same that the one presented in [12] in which we have added a friction law [30] and allowed rotation of the grains.

\subsection{Fluid-grains interaction force}

Only the two dominant components of the fluid-grains interaction force are taken into account, namely the pressure gradient force and the drag force for which there is no fully accepted expression fitting all the flow regimes. Other forces like Basset force, virtual mass effect or the particle lift forces [13] are neglected. The fluid-grain interaction force can be computed at the fluid discretization scale or at the grain scale. The former choice relies on the evaluation of the pressure drop inside a fluid flowing across porous media $[16,21,67]$. In this paper, we will use the latter choice:

$$
\boldsymbol{F}_{s}=-\left.V_{s} \nabla p\right|_{\boldsymbol{x}_{s}}-\gamma\left(\phi, \boldsymbol{x}_{s}, \boldsymbol{u}_{s}, \boldsymbol{u}, a\right)\left(\boldsymbol{u}_{s}-\left.\frac{\boldsymbol{u}}{\phi}\right|_{\boldsymbol{x}_{s}}\right),
$$

where $\gamma$ parametrizes the transition between a linear and a quadratic drag force depending on the particle Reynolds number. Following the argumentation of Richardson and
Zaki [45], the drag force is computed for each grain individually and multiplied by an independent function of the porosity to take into account the influence of the neighbouring grains. The parametrization factor $\gamma$ depends then on the porosity and on a drag coefficient provided by Dallavalle and Klemin [15]:

$$
\begin{aligned}
& \gamma\left(\phi, \boldsymbol{x}_{s}, \boldsymbol{u}_{s}, \boldsymbol{u}, a\right) \\
& =\left.\phi^{-\beta}\right|_{\boldsymbol{x}_{s}} A_{s} \frac{\rho}{2} \\
& \quad\left(0.63\left\|\boldsymbol{u}_{s}-\left.\frac{\boldsymbol{u}}{\phi}\right|_{\boldsymbol{x}_{s}}\right\|^{\frac{1}{2}}+4.8\left[\frac{\mu}{\left.2 r_{s} \rho \phi\right|_{\boldsymbol{x}_{s}}}\right]^{\frac{1}{2}}\right)^{2},
\end{aligned}
$$

where $A_{s}$ is the section area of the grain perpendicular to the flow direction, while the exponent of the simple power law $\beta=1.8$ is given by [66]. More accurate formulas can be found in $[18,32,37,52,71]$. For two-dimensional simulations, the section area $A_{s}$ is simply chosen as the diameter of the grain $s$.

The drag force depending on the fluid density and viscosity is affected by the diffusion of the liquid-gas interface. Particularly, linearly interpolating the dynamic viscosity or the kinematic viscosity has a non-negligible impact on the drag force magnitude near the interface. At fixed fluid volume fraction and relative velocity, the former choice results in a linear evolution of the drag force from its value in the liquid to its value in the gas, while the latter creates an increase in the drag force magnitude near the interface. However, the latter provides a consistent formulation of the one fluid representation with respect to the density interpolation [44] and that is why it is favoured in this work. 
Finally, a grain crossing the interface causes a transfer of the solid volume fraction from one phase to the other one creating phase interchange phenomena that could be significant in some regimes [43]. Different strategies have been suggested to take it into account. It is either possible to modify the evolution equation of the phase indicator function [31], or to change the definition of the phase indicator function itself [43]. The two solutions aim at taking into account the volume occupied by the grains in the mixture but are still limited to dense regimes or introduce a strong variation of the results with the fluid scale [43].

The knowledge about the fluid-grain interaction force is still limited so that there are no fully acceptable solution at this time. We have to keep in mind that the fluid-grain interaction force has a clear impact on the results obtained in CFD-DEM models but studying it is not the subject of this work.

\subsubsection{Terminal velocity of an isolated grain settling in water}

In order to illustrate the model of the fluid-grain interaction, we simulate the fall of a single grain into a fluid. Glass beads $\left(\rho_{s}=2640 \mathrm{~kg} / \mathrm{m}^{3}\right)$ and PVC beads $\left(\rho_{s}=1380 \mathrm{~kg} / \mathrm{m}^{3}\right)$ settling in water $\left(\rho=1000 \mathrm{~kg} / \mathrm{m}^{3}\right.$ and $\left.\mu=10^{-3} \mathrm{~Pa} \mathrm{~s}\right)$ are considered. Terfous et al. [55] have achieved experiments and reported the final velocities of glass and PVC beads in water using different bead radii. The comparison between the numerical results we obtained and the experimental results given by Terfous et al. is presented in Fig. 3. Simulations have been achieved in a box of height $10 \mathrm{~m}$ and width $0.4 \mathrm{~m}$ discretized using elements of size $0.1 \mathrm{~m}$. The settling velocities of the grains have been measured after $1 \mathrm{~s}$ of simulation.

\section{Time integration scheme}

In this section, we develop the time discretization of the equations constituting the multiscale model. The computation of immersed granular flows can be divided into two parts:

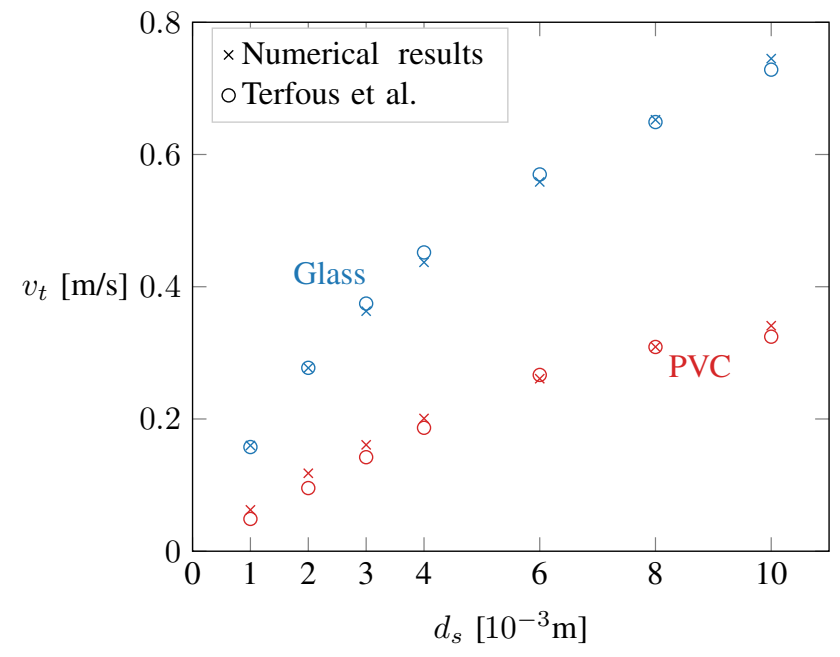

Fig. 3 Comparison between numerical and experimental results for the final settling velocities of glass and PVC beads in water. Cross marks correspond to the experimental results, while the circle marks correspond to the numerical results. In simulation, the recorded terminal velocities correspond to the velocity of the grain after a fall of $1 \mathrm{~s}$

\begin{tabular}{ll}
\hline Fluid solver & $\begin{array}{l}\text { Solve the averaged Navier-Stokes equations to find } \\
\text { the fluid pressure and velocity } \\
\text { Apply the external forces to the grains and solve the } \\
\text { contacts, using the bulk velocity of the mixture }\end{array}$ \\
\hline
\end{tabular}

An explicit coupling is used between the two components so that when computing the evolution of each component the variables coming from the other ones are known. The two solvers, along with the update of the porosity and the phase indicator function, constitute the algorithm computing immersed granular flows at each time step. A predictorcorrector scheme is used for stability reasons. The time loop is presented in Algorithm 1. The decoupling of the fluid and solid solvers results in an algorithm for which the time error is in $\mathcal{O}(\Delta t)$. In this overview, FLUID_SOLVER and GRAINS_SOLVER referred to the fluid and grains solvers that are described hereunder. 


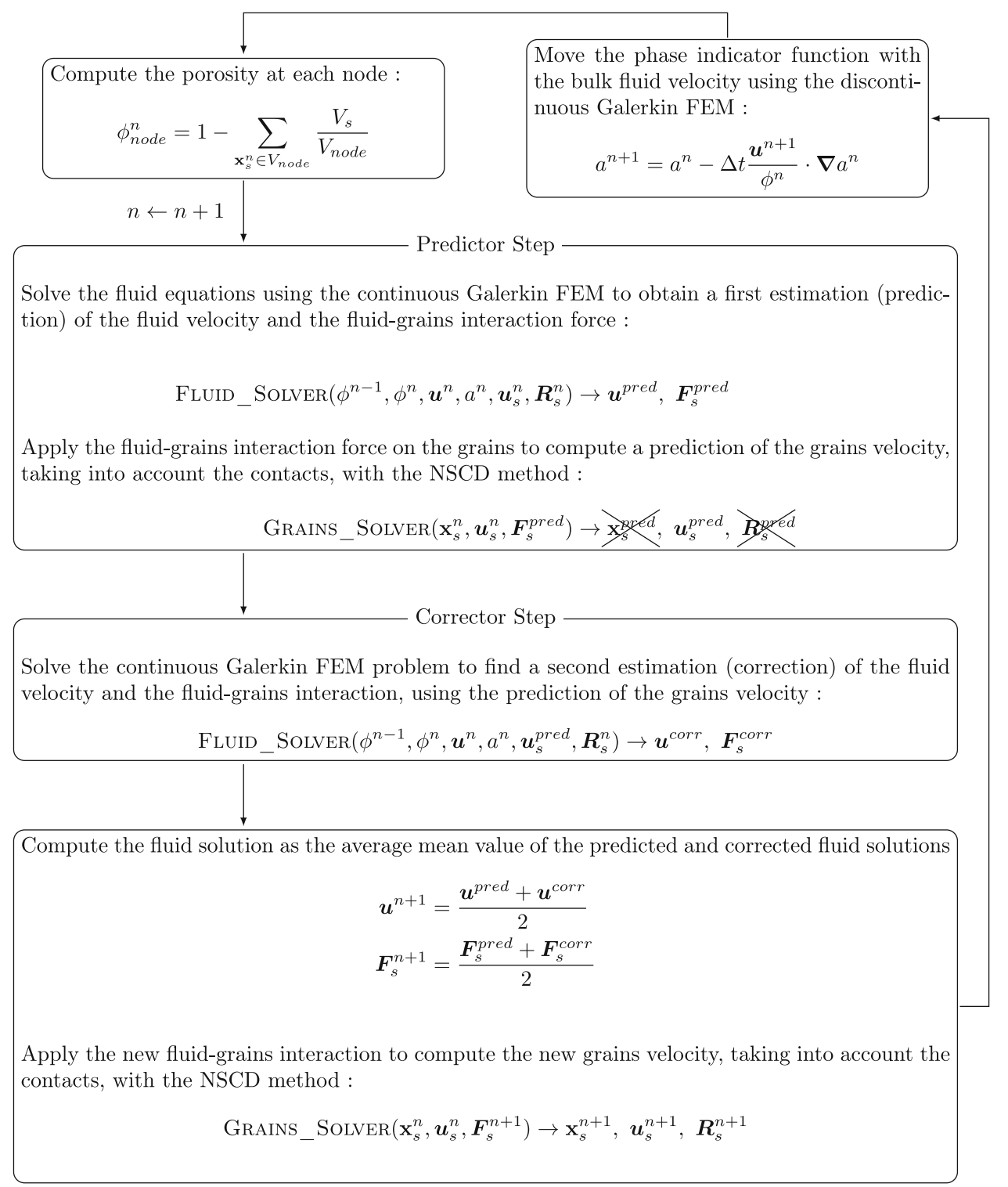

Algorithm 1: Global algorithm used to compute immersed granular flows in time

\subsection{Fluid solver}

At a given time step $n$, the fluid problem is to find $\boldsymbol{u}^{n+1}, p^{n+1}$ such that

$$
\left\{\begin{aligned}
0= & \frac{\phi^{n}-\phi^{n-1}}{\Delta t}+\nabla \cdot \boldsymbol{u}^{n+1}, \\
\rho^{n} \frac{\boldsymbol{u}^{n+1}-\boldsymbol{u}^{n}}{\Delta t}= & -\rho^{n} \nabla \cdot \frac{\boldsymbol{u}^{n+1} \boldsymbol{u}^{n}}{\phi^{n}}+\nabla \cdot 2 \rho^{n} v^{n} \phi^{n} \boldsymbol{d}^{n+1}-\phi^{n} \nabla p^{n+1} \\
& +\phi^{n} \rho^{n} \boldsymbol{g}+\boldsymbol{T}-\sum_{s \in \mathcal{S}}\left(\boldsymbol{F}_{s}^{n+1}-\left.V_{s} \nabla p^{n+1}\right|_{\boldsymbol{x}_{s}^{n}}\right) \delta_{s} \\
\frac{\boldsymbol{u}_{s}^{*}-\boldsymbol{u}_{s}^{n}}{\Delta t}= & \boldsymbol{g}+\frac{1}{m_{s}}\left(\boldsymbol{F}_{s}^{n+1}+\boldsymbol{R}_{s}^{n}\right)
\end{aligned}\right.
$$

where $\delta_{S}$ is the Dirac function at the position of the grain $s$. The last equation of this system is added to explain the semi-implicit scheme used to treat the fluid-grains interaction force.

The explicit coupling makes the fluid-grains interaction force unstable for practicable time steps if treated as a fully explicit term. It is then on purpose to linearize the fluidgrains interaction force to obtain a semi-implicit treatment of this term. This gives us the opportunity to increase the time step by three or four order of magnitude in the air invasion simulations and to consider a time step based on the Courant-Friedrichs-Lewy condition. The fluid-grains inter- 
action force inserted in the above equations should ideally be expressed as:

$$
\begin{aligned}
\boldsymbol{F}_{s}^{n+1}=- & \gamma\left(\phi^{n}, \boldsymbol{x}_{s}^{n}, \boldsymbol{u}_{s}^{n}, \boldsymbol{u}^{n}, a^{n}\right) \\
& \left(\boldsymbol{u}_{s}^{n+1}-\left.\frac{\boldsymbol{u}^{n+1}}{\phi^{n}}\right|_{\boldsymbol{x}_{s}^{n}}\right) \\
& -\left.V_{s} \boldsymbol{\nabla} p^{n+1}\right|_{\boldsymbol{x}_{s}^{n}}
\end{aligned}
$$

However, computing $\boldsymbol{u}_{s}^{n+1}$ along with $\boldsymbol{u}^{n+1}$ requires an implicit coupling between the fluid and the solid parts that is numerically expensive. A prediction $\boldsymbol{u}_{s}^{*}$ of the grains velocity at the next time step is used instead of the exact grains velocity $\boldsymbol{u}_{s}^{n+1}$ so that:

$$
\begin{aligned}
& \boldsymbol{F}_{s}^{n+1}=-\gamma\left(\phi^{n}, \boldsymbol{x}_{s}^{n}, \boldsymbol{u}_{s}^{n}, \boldsymbol{u}^{n}, a^{n}\right) \\
& \left(\boldsymbol{u}_{s}^{*}-\left.\frac{\boldsymbol{u}^{n+1}}{\phi^{n}}\right|_{\boldsymbol{x}_{s}^{n}}\right) \\
& -\left.V_{s} \boldsymbol{\nabla} p^{n+1}\right|_{\boldsymbol{x}_{s}^{n}} .
\end{aligned}
$$

Such a process based on a prediction of the free grains velocity has already been used and proved to be numerically efficient [12] when considering loose grains clusters. In the presence of fully deposed beds of grains, using an approximation of the grains free velocity leads to an underestimation of the drag force applied on the fluid. It is necessary to take into account the forces impeding the movements of the grains like the contact forces between grains or the support reaction for fixed structures when calculating the prediction of the grains velocity. For the sake of concision, these forces are simply represented in the following developments by a resultant reaction force $\boldsymbol{R}_{S}$. The last equation in system (20) is used to compute the prediction of the grains velocity that is introduced in the fluid-grains interaction force. For the sake of concision, system (20) is summarized by:

FLUID_Model $\left(\phi^{n-1}, \phi^{n}, \boldsymbol{u}^{n}, a^{n}, \boldsymbol{u}_{s}^{n}, \boldsymbol{R}_{s}^{n}\right) \rightarrow \boldsymbol{u}^{n+1}, \boldsymbol{F}_{s}^{n+1}$.

\subsubsection{Pressure drop through porous medium}

In the limit of a small Reynolds number, steady incompressible creeping flows are described by the Darcy's law which links linearly the pressure drop through a porous medium to the flow rate across the porous medium:

$-\nabla p=\frac{\mu}{k} \boldsymbol{u}$,

where $k$ is the permeability of the medium. In the context of the Darcy's law, the fluid velocity has to be understood as a volume averaged velocity so that this problem falls within

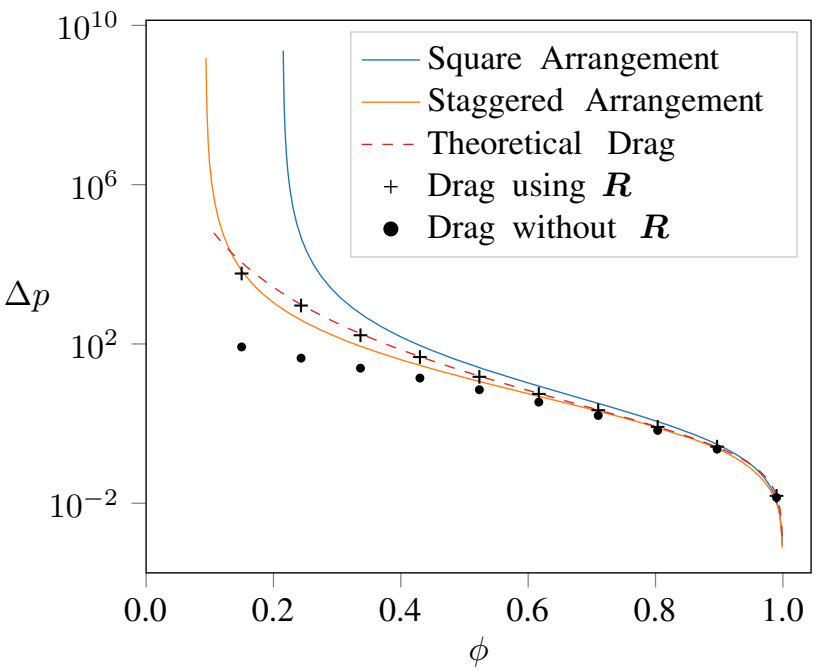

Fig. 4 Pressure drop across a porous medium for $u=10^{-5} \mathrm{~m} / \mathrm{s}$ in the $x$-direction and $\mu=1 \mathrm{~Pa}$. Solid lines show the results obtained using the formulas of Tamayol and Bahrami [54] for a square arrangement (blue) and for a staggered arrangement (orange). Dashed line presents the results obtained by balancing theoretically the drag force given in Eq. (18), and the marks present the results obtained by the use of the complete numerical model. (Color figure online)

the assumption of the multiscale model we developed above. The permeability of the medium must be known a priori. A lot of studies have been devoted to the estimation of the permeability $[25,47,53]$.

Let us consider a fluid with velocity $10^{-5} \mathrm{~m} / \mathrm{s}$ in the $x$ direction crossing a square of side one meter in which grains are placed. Solid lines in Fig. 4 show the pressure gradient given by the formula of Tamayol and Bahrami [54] for specific fibrous arrangements with different volume fractions. In this case, they estimate the permeability as

$k=\frac{0.16 d_{\mathrm{g}}^{2}}{\sqrt{\phi}}\left(3+\frac{1-\phi_{m}}{1-\phi}-3 \sqrt{\frac{1-\phi_{m}}{1-\phi}}-\sqrt{\frac{1-\phi}{1-\phi_{m}}}\right)$,

where

$\phi_{m}= \begin{cases}1-\frac{\pi}{4} & \text { Square arrangement } \\ 1-\frac{\pi}{2 \sqrt{3}} & \text { Staggered arrangement }\end{cases}$

is the minimum fluid volume fraction a particular arrangement can provide.

The arrangement is not taken into account in the drag formula (18) we chose to parametrize the viscous fluid-grains interaction. The dashed line shown in Fig. 4 corresponds to the pressure gradient that theoretically balances this drag force. It provides results lying in the range of the results obtained by Tamayol and Bahrami [54]. This red dashed 
curve constitutes the best result we could expect if the numerical method was perfectly accurate without any dissipation. Discretizing the square with elements ten times smaller than the square side, our model can be used to compute the flow crossing randomly placed grains. The pressure gradient we obtain numerically is shown in Fig. 4. In the case of important solid constraints, neglecting the reaction forces $\boldsymbol{R}$ (dot marks) leads to an underestimation of the drag force because the prediction provides a nonzero grain velocity that is inconsistent with the grains mobility. Taking into account the reaction force $\boldsymbol{R}$ when predicting the grains velocity is then mandatory for small porosities (plus marks).

\subsection{Grains solver}

As we use an explicit coupling between the solid and fluid motions, when computing the velocity and position of the grains, all the forces applied on the grains are known except the reaction forces exerted on a grain by its neighbours. Considering grains as Lagrangian solid and non-deformable particles, it is required to treat the contacts between each pair of grains and between the grains and the boundaries. The NSCD method [30] has been created to compute the velocities of a set of grains constrained by the others and the boundaries without interpenetrations. The update of the grains position is obtained using the grains velocity $\boldsymbol{u}_{s}^{n+1}$ :

$\boldsymbol{x}_{s}^{n+1}=\boldsymbol{x}_{s}^{n}+\Delta t \boldsymbol{u}_{s}^{n+1}$.

This set of velocities is computed from the second Newton's law of motion:

$\boldsymbol{u}_{s}^{n+1}=\boldsymbol{u}_{s}^{n}+\frac{\Delta t}{m_{s}}\left(m_{s} \boldsymbol{g}+\boldsymbol{F}_{s}^{n+1}+\boldsymbol{R}_{s}^{n+1}\right)$,

where the total reaction force $\boldsymbol{R}_{s}^{n+1}$ applied on the grain $s$ is the sum of the reaction forces exerted by the other grains $j$ :

$\boldsymbol{R}_{s}^{n+1}=\sum_{j \in\{\mathcal{S} \backslash s\}} \boldsymbol{R}_{s j}^{n+1}$.

The contributions of the boundaries to the reaction are not detailed because they are treated in the same way than the grain-grain interaction forces. These grain-grain reaction forces are computed such that their normal component respects:

the impenetrability condition:

$$
0 \leq\left\|\mathbf{x}_{s}^{n+1}-\mathbf{x}_{j}^{n+1}\right\|-\left(r_{s}+r_{j}\right), \quad \forall j \in\{\mathcal{S} \backslash s\},
$$

the no attraction condition:

$$
0 \leq \boldsymbol{R}_{s j}^{n+1} \cdot \mathbf{n}_{s j}, \quad \forall j \in\{\mathcal{S} \backslash s\},
$$

and such that these reaction forces only act between grains that are in contact:

$$
\begin{gathered}
0=\left[\left\|\mathbf{x}_{s}^{n+1}-\mathbf{x}_{j}^{n+1}\right\|-\left(r_{s}+r_{j}\right)\right] \\
\boldsymbol{R}_{s j}^{n+1} \cdot \mathbf{n}_{s j}, \quad \forall j \in\{\mathcal{S} \backslash s\} .
\end{gathered}
$$

These conditions are sufficient to ensure that there are no overlapping between grains. In the case of grain-grain reaction force with a strictly positive normal component, its tangential component is computed to respect the Coulomb's law of friction. If the tangential part of the relative velocity of the two contacting grains is not zero after the application of the normal component of the contact reaction forces, there are two different possible cases. Either the tangential component of the reaction force resulting from this tangential relative velocity is small compared to its normal component. In this case, the reaction forces must be computed such that their tangential component cancels the tangential relative velocity. This corresponds to a non-slip condition in which the threshold on the tangential component of the contact reaction is determined through the dynamic friction coefficient $\mu_{s j}$. In the other case, the magnitude of the tangential component of the reaction forces is determined by its normal component and acts in the opposite direction of the tangential relative velocity:

$\left\|\boldsymbol{R}_{s j}^{n+1} \cdot \mathbf{t}_{s j}\right\|=\max \left(\mu_{s j} \boldsymbol{R}_{s j}^{n+1} \cdot \mathbf{n}_{s j}, R_{t}^{*}\right)$,

where $\mathbf{n}_{s j}$ and $\mathbf{t}_{s j}$ are, respectively, the unit centre-to-centre normal vector and the unit tangential vector, while $R_{t}^{*}$ is computed such that

$\left(\boldsymbol{u}_{s}^{n+1}-\boldsymbol{u}_{j}^{n+1}\right) \cdot \mathbf{t}_{s j}=0 \quad \forall j \in\{\mathcal{S} \backslash s\}$

The directions of $\boldsymbol{R}_{s j}^{n+1} \cdot \mathbf{t}_{s j}$ and $R_{t}^{*}$ are the same. A similar algorithm to the one described in [12] is used to find the reaction forces and the grains velocity respecting the above conditions. The friction constraints have been inserted in this algorithm to compute the grains angular velocity based on the tangential component of the reaction forces. It has to be noted that this algorithm is based on a perfectly inelastic contact law so that there is no restitution between grains during a collision. Similarly to what has been done for the fluid part, this system is summarized by:

GRAINS_SOlver $\left(\mathbf{x}_{s}^{n}, \boldsymbol{u}_{s}^{n}, \boldsymbol{F}_{s}^{n+1}\right) \rightarrow \mathbf{x}_{s}^{n+1}, \boldsymbol{u}_{s}^{n+1}, \boldsymbol{R}_{s}^{n+1}$. 
Fig. 5 Initial situation for the experimental set-up. Settled polystyrene grains form a bed of height $h_{\mathrm{g}}$ in ethanol. The height of fluid above the bed is $h_{l}$. Air is injected at constant inflow rate $I=6.5 \mathrm{~mL} / \mathrm{min}$ in the injection pipe where the pressure $p_{\text {in }}$ is measured. $\Omega_{M}$ is injector in which motion of grains is considered the area centred around the

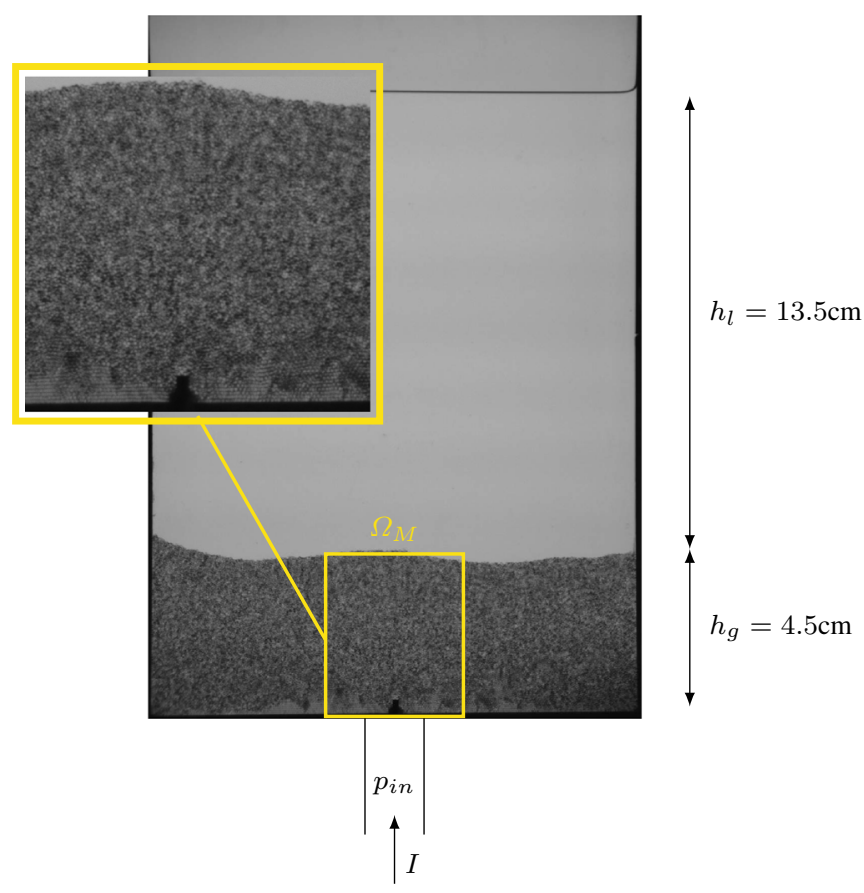

The results achieved with $h_{\mathrm{g}}=9 \mathrm{~cm}$ are used to compare the invasion process of the granular bed for different inflow rates.

Having demonstrated the ability of the model to reproduce simplified problems, we are now able to face the objective of this paper, which is the simulation of air invasion in immersed granular beds. Specifically, this section aims to present the ability of the model to reproduce the beginning of the invasion process while giving insight in the mechanical properties of the granular bed. Experiments have been achieved in a HeleShaw cell to compare to the numerical work.

\subsection{Experimental set-up}

Initially, a granular bed of height $h_{\mathrm{g}}$ is deposited in the cell filled with fluid. The height, width and gap $e$ of the cell are, respectively, $29.3 \mathrm{~cm}, 13.6 \mathrm{~cm}$ and $e=0.2 \mathrm{~cm}$. The height of fluid above the granular surface is denoted $h_{l}$. The initial condition is obtained by the sedimentation of a set of grains in the fluid. A mass-flow controller (Bronkhorst, Mass-Stream Series D-5111) is connected to an injector of external diameter $d_{\text {in }}=2 \mathrm{~mm}$ at the bottom of the cell via a plastic pipe. Grains are polystyrene spherical beads ( $\rho_{s}=1059 \mathrm{~kg} / \mathrm{m}^{3}$ and $\left.d_{s}=500-630 \mu \mathrm{m}\right)$ and the fluid is ethanol $\left(\rho=785 \mathrm{~kg} / \mathrm{m}^{3}\right.$ and $\left.\mu=1.2 \times 10^{-3} \mathrm{~Pa} \mathrm{~s}\right)$. Images acquisition is achieved at constant rate using a camera PixeLink PL B781U. Two heights of granular bed are considered below. The results obtained with $h_{\mathrm{g}}=4.5 \mathrm{~cm}$ are used to calibrate the parameters of the model and to describe the relevant inflow condition to consider in the simulation.

\subsection{Experimental observations about the system dynamics at short times}

Let us consider the situation depicted in Fig. 5 where air is injected at constant inflow rate $I=6.5 \mathrm{~mL} / \mathrm{min}$ in a granular bed of height $h_{\mathrm{g}}=4.5 \mathrm{~cm}$. The mass-flow controller regulating the inflow rate is not plugged directly at the cell injector due to experimental constraints but through a flexible pipe. It acts as a chamber into which the gas accumulates until the pressure in the chamber is high enough to push the gas through the granular bed. The differential pressure in the injection pipe is measured using a pressure sensor FGP Instrumentation P211 and presented in Fig. 6. In the experiments, the reference pressure $p_{0}$ is the atmospheric pressure. Initially, the pressure increases in the pipe until a maximum value and then decreases rapidly before increasing again and repeating the same behaviour. These oscillations in the pressure are due to the intermittent air invasion of the granular bed. At the beginning, the pressure increases in the injection pipe until the gas is able to penetrate the granular layer, either by percolation or, in this experiment, by fracturing the bed (see image in Fig. 10). As soon as the cavity or the channel is large enough to reach the fluid-grains interface, the air escapes from the granular bed in the form of bubbles. 


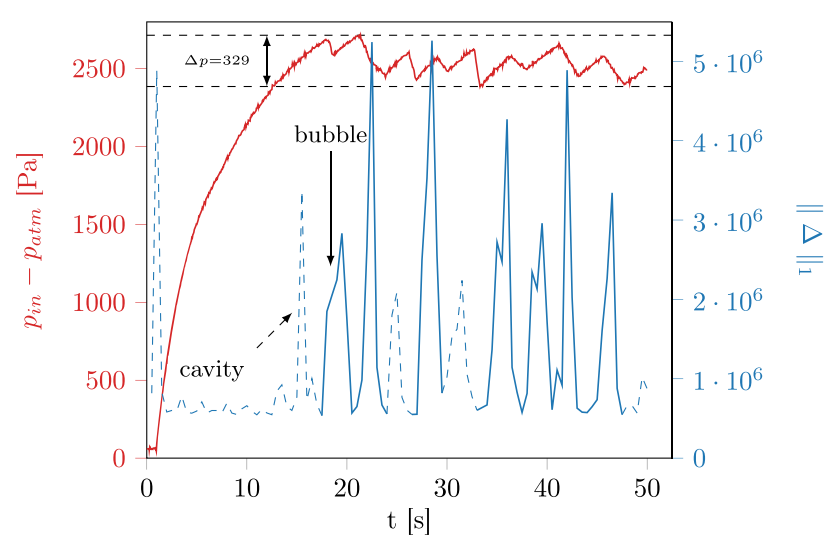

Fig. 6 Evolution of the differential pressure in the injection pipe and Manhattan norm of the difference between pixels of two successive image captures situating in time important motions in the granular bed for the case $h_{\mathrm{g}}=4: 5 \mathrm{~cm}$ and $I=6: 5 \mathrm{~mL} / \mathrm{min}$. $p_{\text {atm }}$ is the atmospheric pressure. Dashed parts of the Manhattan norm curve represent the movements that are not associated with a bubble emission

The Manhattan norm of the difference $\Delta$ between pixels of two successive image captures in the $\Omega_{M}$ area centred around the injector is computed to quantify the motion of the grains in the nearby area and shown side by side with the pressure in the injection pipe (Fig. 6). It can be seen that there are more peaks in the motion curve than in the pressure curve so that the correlation might not be evident without insights in the experiment evolution. A motion in the granular matrix is not necessarily related to a bubble emission so that we have to identify the corresponding peaks in the motion curve. Figure 7 shows the peaks in the motion curve with the experimental observations. During the first peak, as the pressure increases, the force is sufficient to spread the grains around the injector, but not to push the air up to the fluid-grains interface. At this time, the cavity stays open for a moment without any motion of the grains. The pressure continues to accumulate inside the cavity until it is sufficiently high to break the equilibrium state and to push the air towards the fluid-grains interface where a bubble is emitted. Figure 7 also shows that a peak in the motion curve can be related to two successive bubble emissions. Sometimes, air is trapped in a cavity created in the wake of a bubble. If a large quantity of air is trapped, a small increase in pressure, i.e. a small additional amount of air, is sufficient to create a second bubble just after the first one. In the experiment, it happens after about $27 \mathrm{~s}$. A last clarification concerns the increase in $\|\Delta\|_{1}$ observed between 0 and $1 \mathrm{~s}$ that is due to a residual cavity around the injector that flows back in the injection pipe at the valve opening. These considerations give us the opportunity to separate in Fig. 6 the peaks in the motion curve related to a bubble emission (solid line) from the other ones (dashed line).

The phases of a common period are shown in Fig. 8. The first phase consists of the increase in the pressure in the injec-

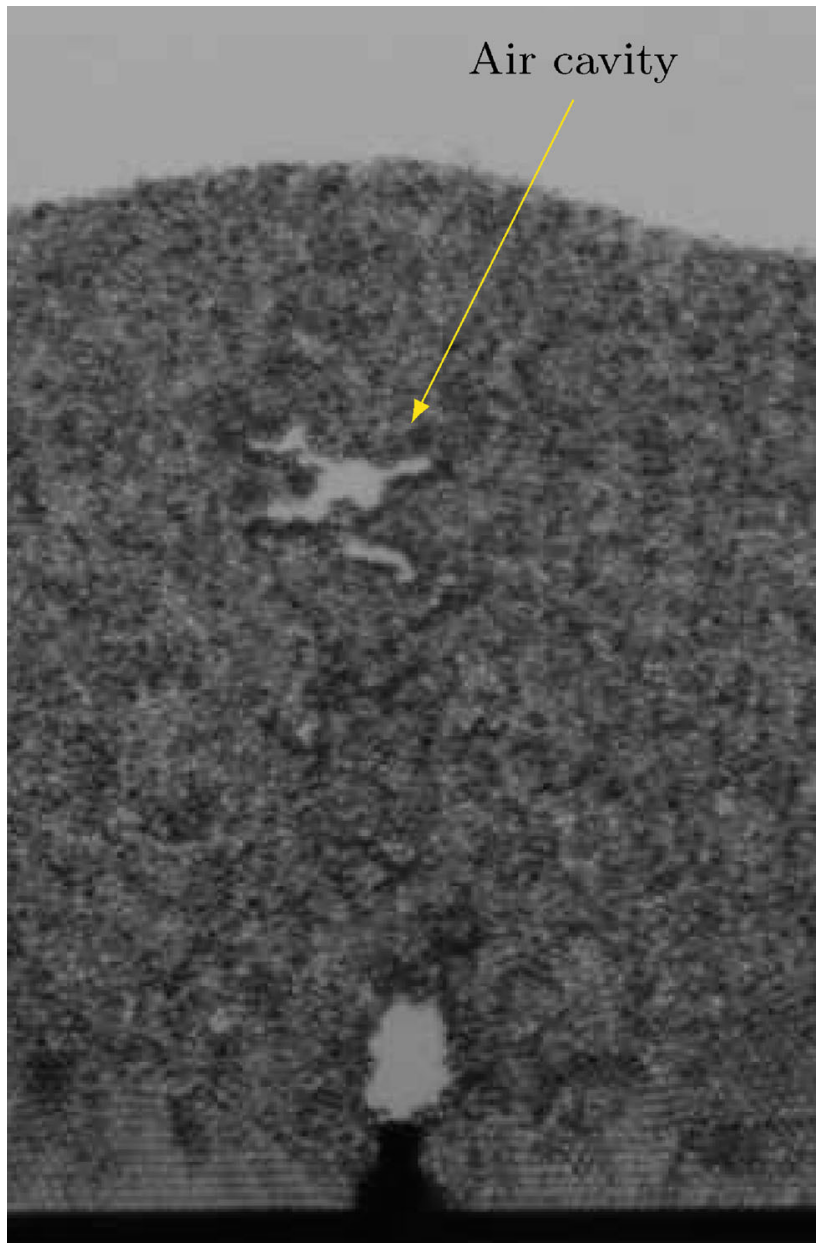

Fig. 7 Granular bed fracture due to air invasion creates cavities in which air may be clogged

tion pipe, nothing being visible in the granular bed. Then, the pressurized gas spreads the grains creating a small cavity around the injector. The gas ascends through intricate channels towards the grains-fluid interface where it forms a bubble. The upward motion of the bubble is sufficient to entrain some grains in its wake so that the fluid-grains interface is modified by successive transports and depositions of the grains (Fig. 9).

\subsection{Numerical simulations}

Setting the right boundary conditions in the multiscale model is a critical point to compute accurately the experiment. The inflow boundary condition has to be chosen in order to reproduce a pressure signal similar to the one observed in the experiment. This choice is presented in the following part, along with a modelling of viscous effect of the front and rear wall of the Hele-Shaw cell in the twodimensional simulations. The numerical parameters are then validated on the experiments and results are investigated. 

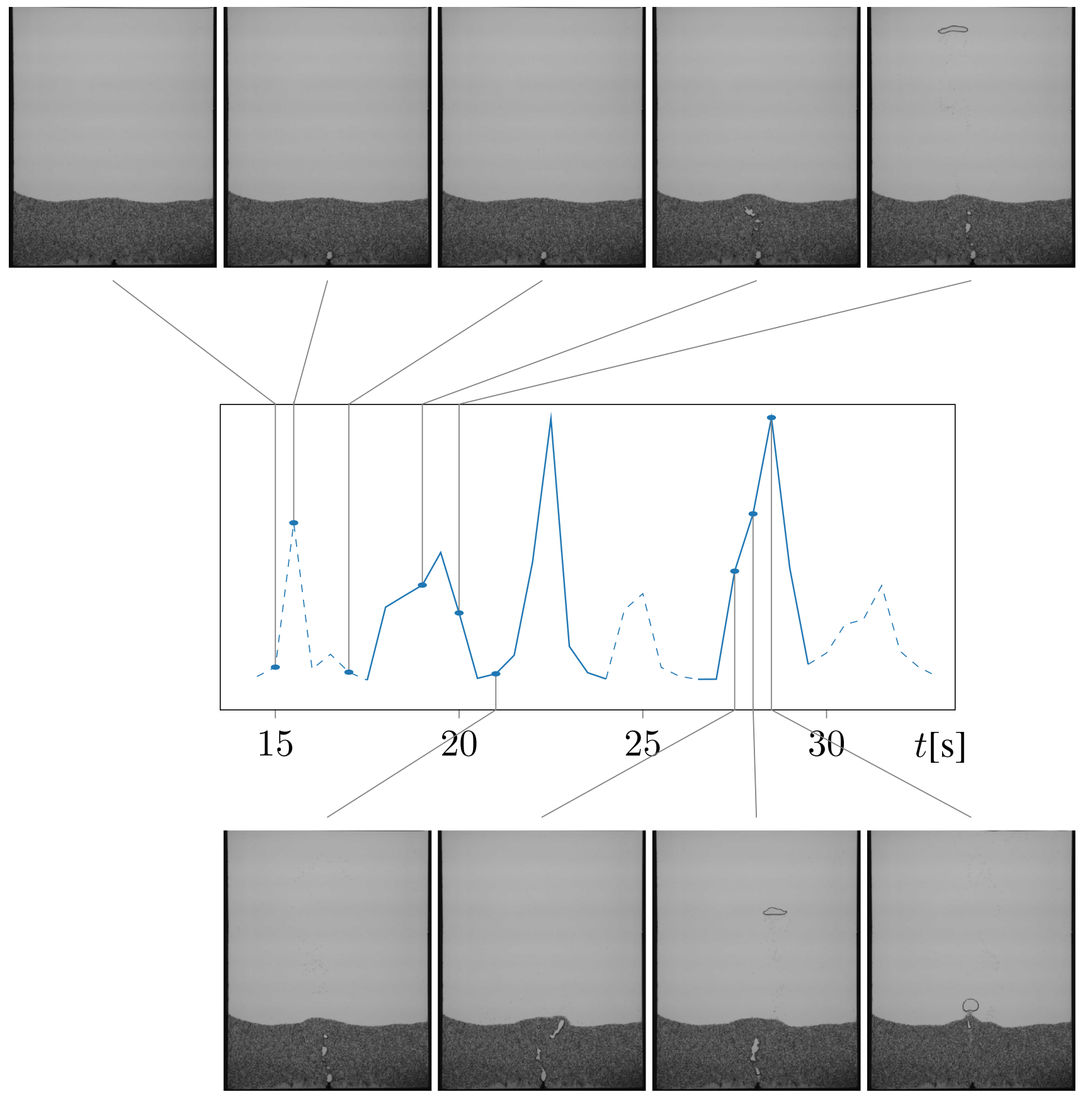

Fig. 8 Successive events can be related to motions in the granular bed. Events are located in time on the difference curve used to determine a motion during the experiment. Some peaks are related to a cavity cre- ation without bubble emission (top images), while two bubbles can be created during the same motion peak (bottom images)

tion scale as averaging scale requires to use elements that are three times larger than the grain diameter [65]. For all the simulations, the computational domain consists in an unstructured grid of triangles uniformly refined of size $h=1.5 \mathrm{~mm}$ so that the element size-to-grains diameter ratio is approximately equal to 3 . This choice allows to represent the injection area and provides a large-scale representation of
In this work, we only consider two-dimensional simulations of the air invasion process. The smaller pile consists in 20,959 grains with a diameter uniformly distributed in the range $500<d_{s}<600 \mu \mathrm{m}$. The grain diameter-to-cell depth ratio is approximately equal to 4 . In addition to the computational cost required to compute three-dimensional simulations ( $>80,000$ grains), the use of the fluid discretiza- 
the porosity in order to prevent considerable mistakes [40]. As a result, the flow should have been discretized with less than two elements in depth giving an irrelevant description of the flow.

\subsubsection{Inflow boundary condition}

Due to the element size-to-grains diameter ratio, it is not possible to accurately represent the injector shape. The injection area is considered horizontal and at the level of the bottom of the cell. It is considered as a rectangle area of width $d_{\text {in }}$ and its fictional depth for the computation of the inflow rate is $e$.

The analysis of the experiments shows that the boundary condition at the injection aperture is not a simple constant inflow rate. The compressibility of the gas in the injection chamber must be taken into account by setting a special pressure boundary condition on the edges constituting the injection surface. The injected air is considered as an ideal gas and the injection pipe volume as constant. The ratio between the pressure $p_{\text {in }}$ and the mass of gas $m_{\text {in }}$ inside the injection pipe is:

$\frac{p_{\text {in }}(0)}{m_{\text {in }}(0)}=\frac{p_{\text {in }}(t)}{m_{\text {in }}(t)}$.

The gas mass flux $I$ entering the injection pipe is constant and driven by the mass-flow controller. The pressure in the injector boundary condition is obtained from Eq. (31), while the pressure in the computational domain along this boundary is known from the computation. The mass flux through the interface $S_{\text {in }}$ between the injection pipe and the Hele-Shaw cell is computed by balancing these two pressures:

$$
\frac{\mathrm{d} m_{\mathrm{in}}(t)}{\mathrm{d} t}=\rho_{\mathrm{g}}\left(I+e \oint_{S_{\mathrm{in}}} \boldsymbol{u} \cdot \boldsymbol{n} \mathrm{d} S\right)
$$

where $\rho_{\mathrm{g}}$ is the air density, $\boldsymbol{u}$ is the fluid velocity in the cell computed with the model and $\boldsymbol{n}$ is the unit normal to the boundary. Simulations are achieved using the initial condition:

$m_{\text {in }}(0)=V_{\text {in }}(0) \rho_{\mathrm{g}}$,

where $V_{\text {in }}$ is the volume of the injector pipe and is a flexible parameter of the model. A force is imposed at the top open boundary to constrain the pressure at a zero value. The initial value of the pressure $p_{\text {in }}(0)$ in the injection pipe is tuned to avoid the long pressure increase period at the start of the experiment observed in Fig. 6.

\subsubsection{Effect of front and rear walls}

The front and rear walls of the Hele-Shaw cell impact the behaviour of the experiment. The objective is to reproduce the experiment by achieving a bidimensional simulation in which the front and rear walls are not considered. It is then not possible to represent explicitly the viscous effects damping the fluid flow in the vicinity of these walls by prescribing a no-slip boundary condition as it is done for the other walls. This effect is modelled through a volume drag force applied on the fluid. This force reads

$\boldsymbol{F}_{w}=D \mu \boldsymbol{u}$

and is inserted into the fluid momentum Eq. (2). This adds a new coefficient $D$ that needs to be calibrated.

In this work, we use the experiment achieved with the lowest inflow rate $I=6.5 \mathrm{~mL} / \mathrm{min}$ and the smallest height of grains $h_{\mathrm{g}}=4.5 \mathrm{~cm}$ to calibrate the simulation. Three parameters are calibrated to match the experimental and numerical results: the surface tension coefficient $\sigma$, the volume of the injection chamber $V_{\text {in }}$ and the coefficient of the volume drag $D$ due to the front and rear walls. The surface tension coefficient has been varied around its real value for air-ethanol flows $\sigma=22.39 \times 10^{-3} \mathrm{~N} / \mathrm{m}$ until we reach an optimal value of $\sigma=0.06 \mathrm{~N} / \mathrm{m}$. The difference can be explained by the loss of a dimension and the coarse resolution scale. For the ascent of a bubble from the bottom to the top of the cell, this gives a Bond number

Bo $=\frac{g L^{2} \Delta \rho}{\sigma}=\frac{9.81 \cdot 0.18^{2}(785.92-1.117)}{0.06}=4157.42$.

Using the bubbles size, the optimal value for the volume of the injection pipe is $V_{\text {in }}=240 \mathrm{~cm}^{3}$. This value has the expected order of magnitude compared to the experiment in which a pipe of diameter $\sim 1 \mathrm{~cm}$ and length $\sim 1 \mathrm{~m}$ has been used. The starting point for the calibration of the drag coefficient $D$ is the Hagen-Poiseuille equation. Considering a Hagen-Poiseuille flow between two plates in the transverse direction, the vertical pressure drop in the fluid is:

$$
\frac{\mathrm{d} p}{\mathrm{~d} y}=12 \frac{\mu}{h^{2}} \overline{\boldsymbol{v}} \approx D \mu \boldsymbol{u}
$$

where $\bar{v}$ is the depth-averaged velocity and $h$ is the element size. This gives a coefficient $D=1.92 \times 10^{6} \mathrm{~m}^{-2}$. Based on the ascent velocity of the bubble, the estimated optimal value is $D=2.5 \times 10^{6} \mathrm{~m}^{-2}$. All these parameters have been calibrated considering a static friction coefficient $\mu_{s}=0.4$. The friction coefficient could be a source of mistakes in the following computations because its value is not well known for lubricated spherical grains. However, we could expect 


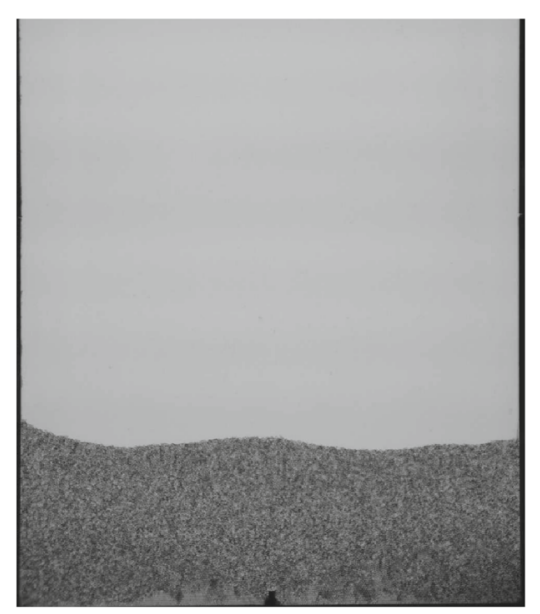

$t=15 \mathrm{~s}$

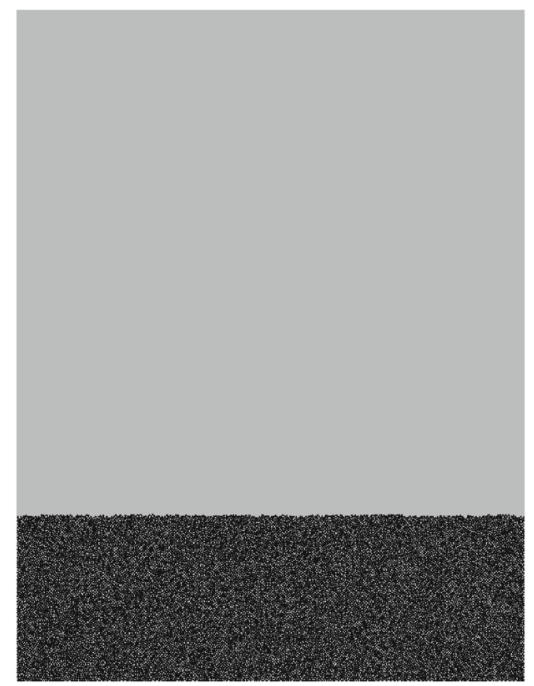

$t=15 \mathrm{~s}$
Experiment

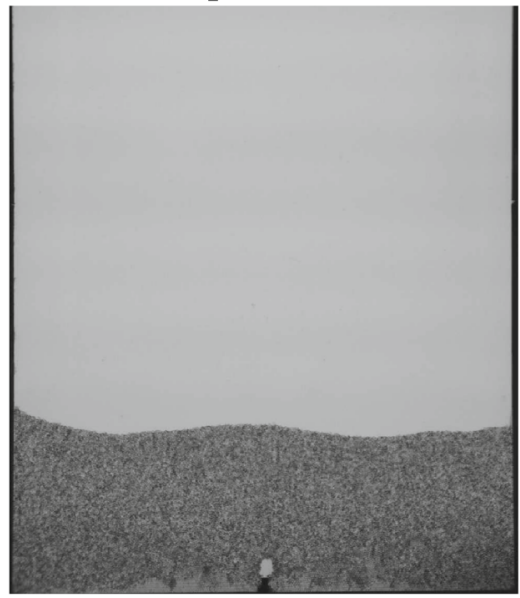

$t=17.5 \mathrm{~s}$

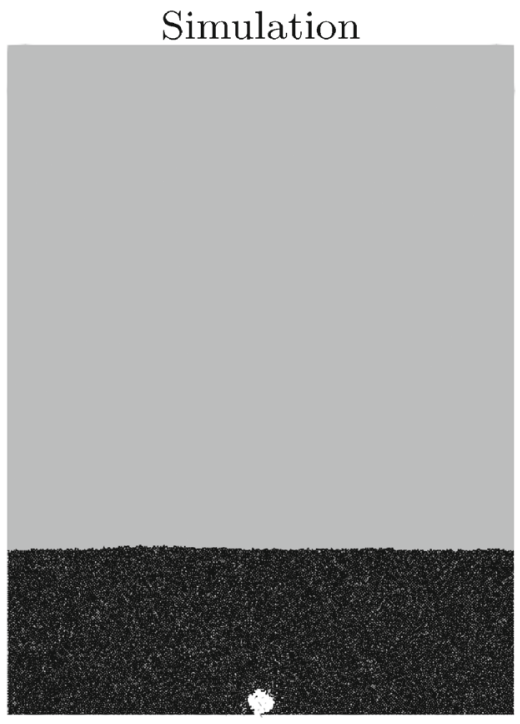

$t=17.5 \mathrm{~s}$

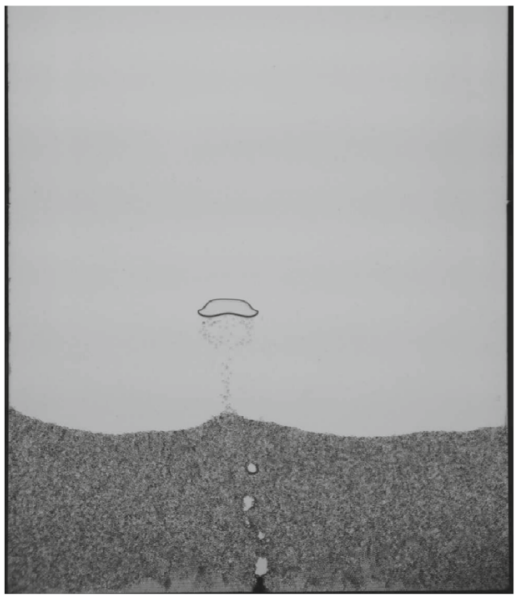

$t=19.5 \mathrm{~s}$

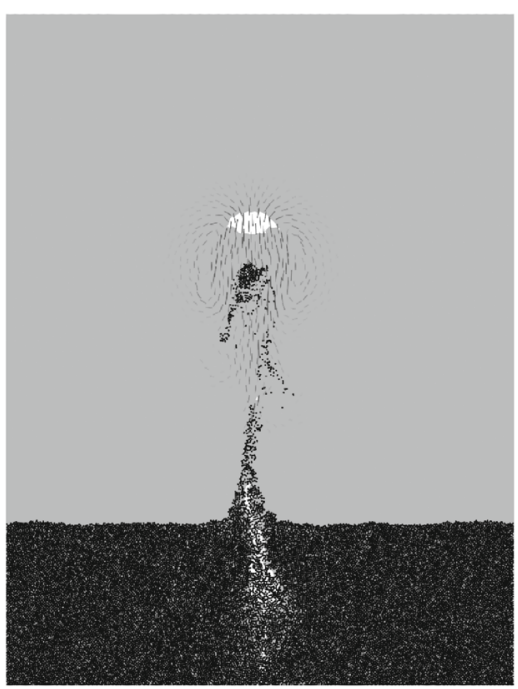

$t=19.5 \mathrm{~s}$
Fig. 9 Comparison between experimental and numerical results shows the system evolution during one period of gas invasion for $h_{\mathrm{g}}=4.5 \mathrm{~cm}$ and $I=6.5 \mathrm{~mL} / \mathrm{min}$. The left figures show the initial bed, the middle ones the cavity creation as the pressure increases in the pipe and the right ones a bubble ascent with grains in its wake

$I=6.5 \mathrm{~mL} / \mathrm{min}$. The significance of the capillary forces with respect to the viscous forces is characterized by the capillary number:

$\mathrm{Ca}=\frac{\mu \boldsymbol{u}_{m}}{\sigma}=\frac{1.2 \times 10^{-3} \cdot 0.4}{0.06}=8 \times 10^{-3}$,

\subsubsection{Validation of the numerical results based on the pressure signal and bubble sizes}

Figure 8 shows the comparison between the experimental and numerical results of air invading a bed of height $h_{\mathrm{g}}=4.5 \mathrm{~cm}$ constituted of polystyrene spherical beads with an inflow rate where $\boldsymbol{u}_{m}$ is the mean velocity of the successive bubbles at the middle height of the initial deposit. The choice of $\boldsymbol{u}_{m}$ as a characteristic velocity of the flow is made because we are mainly interested in the flow dynamics in the deposit. The 
particle Reynolds number associated with $\boldsymbol{u}_{m}$ is:

$\mathrm{Re}_{s}=\frac{\rho \boldsymbol{u}_{m} d_{s}}{\mu}=\frac{785 \cdot 0.4 \cdot 0.0005}{1.2 \times 10^{-3}}=130.8$,

characterizing a flow without turbulent effects.

The fluid time step is computed so that, during it, the fluid flow does not cross more than the radius $R_{h}$ of the incircles of the mesh triangles:

$\Delta t=\frac{R_{h}}{4 \boldsymbol{u}_{\max }}=\frac{7.2 \times 10^{-4}}{2.8} \mathrm{~s}=2.5714 \times 10^{-4} \mathrm{~s} \approx 2.5 \times 10^{-4} \mathrm{~s}$,

where $\boldsymbol{u}_{\max }$ is the maximum fluid velocity determined numerically for $I=6.5 \mathrm{~mL} / \mathrm{min}$ and factor 4 is used as a safety margin. A similar analysis has been made to determine the time step of the other cases. The solid time step is five times smaller than the fluid one for each simulation. The factor five has been determined empirically to minimize the computational cost as a trade-off between the number of subtime steps (each requiring programmes such as the colliding pair searching) and the number of collisions to handle by the contact solver at each sub-time step which is decreased for smaller sub-time steps.

After calibration, the model shows the same behaviour than the experimental results. The pressure increases at the injection boundaries due to the ideal gas law and the constraints caused by the bed of grains and the surface tension. As soon as the pressure reaches a critical value, air is pushed inside the box and starts its ascent through the granular medium. Figure 8 shows that the air bubble carries grains in its wake after leaving the granular bed. Then, the pressure at the injection boundary decreases with the release of the gas inside the box. The grains spread by the air fall and regain their position, as the fluid does, clogging again the air inlet. The pressure increases again and so on.

Figure 11 shows the cycle of pressure variations and bubble creations that are linked to the displacement $\Delta x_{s}$ of each grain during the simulation. The higher value of the first pressure peak with respect to the following ones can be explained by the initial arrangement of the grains in the bed. The simulation is started using a compact arrangement that is hard to break due to friction between grains. Once the first air bubble has gone through the granular bed, sedimentation creates loose packing above the air inlet. It facilitates the invasion of the next bubbles. It can be seen that the order of magnitude of the pressure variations $\Delta p$ during one cycle is the same as the one observed in the experiment. Numerical results can also be validated by comparing the properties of the system. Table 1 shows the comparison of the mean time step $\bar{T}$ between two pressure peaks and the mean diameter $\bar{D}$ of the bubbles. This diameter has been measured at a height of 0.07

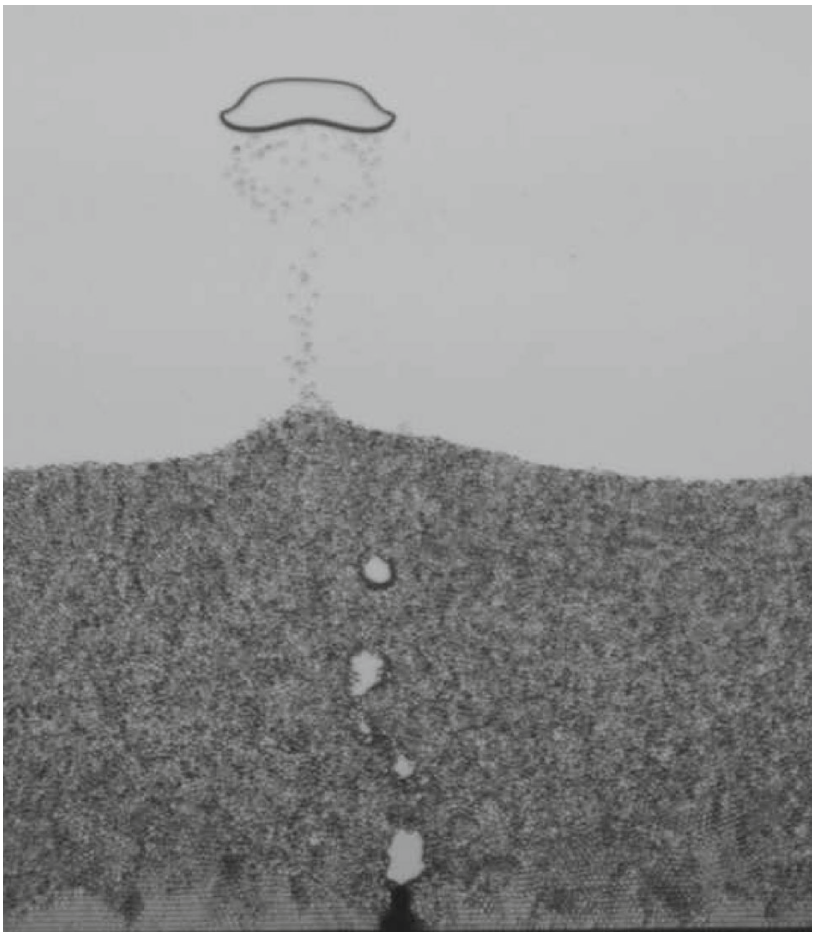

Fig. 10 Bubble carrying on grains in its wake for $I=6.5 \mathrm{~mL} / \mathrm{min}$ and $h_{\mathrm{g}}=4.5 \mathrm{~cm}$ at $t=19.5 \mathrm{~s}$

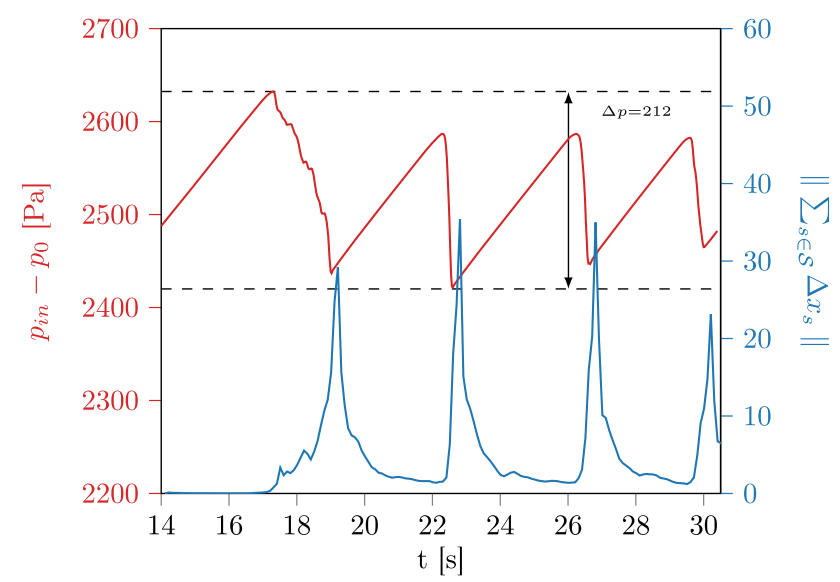

Fig. 11 Simulated evolution of the differential pressure in the injection pipe and norm of the displacement of all grains in time for the case $h_{\mathrm{g}}=4.5 \mathrm{~cm}$ and $I=6: 5 \mathrm{~mL} / \mathrm{min}\left(\mathrm{Ca}=8 \times 10^{-3}, \mathrm{Re}=130.8\right.$ and $\left.\Delta t=2.5 \times 10^{-4} \mathrm{~s}\right) . p_{0}$ is the reference pressure

$\mathrm{m}$ from the bottom of the cell so that the mean process is not biased by the numerical diffusion during the bubble ascent.

Having calibrated the numerical parameters on the experiment with the lowest inflow rate $I=6.5 \mathrm{~mL} / \mathrm{min}$, it is normal to find similarities between the experiment and the simulation. The model has been validated by changing the inflow rate and by comparing the experimental and numerical results. In a second experimental set-up, we keep the same height $h_{\mathrm{g}}=4.5 \mathrm{~cm}$ of polystyrene beads in ethanol but 
Table 1 Comparison between the mean time separating two pressure peaks and the mean diameter of the successive bubbles at a height of $0.07 \mathrm{~m}$ for the experiments and the simulations using $h_{\mathrm{g}}=4.5 \mathrm{~cm}$

\begin{tabular}{llllll}
\hline \multirow{2}{*}{ smallskip } & \multicolumn{2}{l}{$I=6.5 \mathrm{~mL} / \mathrm{min}$} & & \multicolumn{1}{l}{$I=13 \mathrm{~mL} / \mathrm{min}$} & \\
\cline { 2 - 3 } & $\bar{T}(\mathrm{~s})$ & $\bar{D}(\mathrm{~cm})$ & & $\bar{T}(\mathrm{~s})$ & $\bar{D}(\mathrm{~cm})$ \\
\hline Experiment & $3.22 \pm 0.52$ & $2.03 \pm 0.35$ & & $1.72 \pm 0.55$ & $1.86 \pm 0.27$ \\
Simulation & $3.32 \pm 0.49$ & $1.60 \pm 0.30$ & & $2.51 \pm 0.42$ & $1.87 \pm 0.42$ \\
\hline
\end{tabular}

with a double inflow rate $I=13 \mathrm{~mL} / \mathrm{min}$. The data analysis (Table 1) shows that the mean diameter of bubbles are similar in the experiment and in the simulation. The mean time step between two pressure peaks is close, but the results show that it is overestimated in the simulation. A possible explanation for this phenomenon can be attributed to the averaging process and the coarse fluid scale. As it can be observed on the experiment with the lower inflow rate, it happens that the air clogged in a cavity takes advantage of the ascent of a bubble to escape from the granular bed. It then creates secondary bubbles in the wake of the first ones. Cavities cannot be observed in the simulation because the fluid spatial discretization is too coarse to have an accurate representation of the ethanol-air interface in the granular bed. The higher the inflow rate, the more the granular bed moves. It possibly increases the secondary bubble occurrences appearing in the experimental pressure signal. The period of the numerical pressure signal may then be increased because the simulation only represents the main events that have a scale large enough to be captured. This speculation requires more highfrequency observations of the experimental air invasion at short times to be validated. Finally, comparison of the differential pressure signals is given in Fig. 12 to show that the magnitude of the pressure variations is quite the same in each case.

\subsubsection{Fluidized area evolution}

The fluidized area morphology has been carefully considered in previous work $[33,62]$. It has been shown that the fluidized area starts at the top of the bed because the grains are able to move freely while they are constrained in the bed. Then, the fluidized area grows from a cone shape to an area that can be fitted with a logarithmic law. However, the actual motion of the grains during the fluidization process is not accessible through the experiments. Figure 13 shows the comparison between the areas where the movements of the grains are the most important in the experiment and in the simulation. The motion areas are concentrated near the centre along the channels created by the bubbles. The channels created by the bubbles are obviously the areas where the motion is the highest, but Fig. 13 suggests that the grains around the channels are involved in the global dynamics of the bed. It shows the number of grains that have to be moved to enable the air invasion.

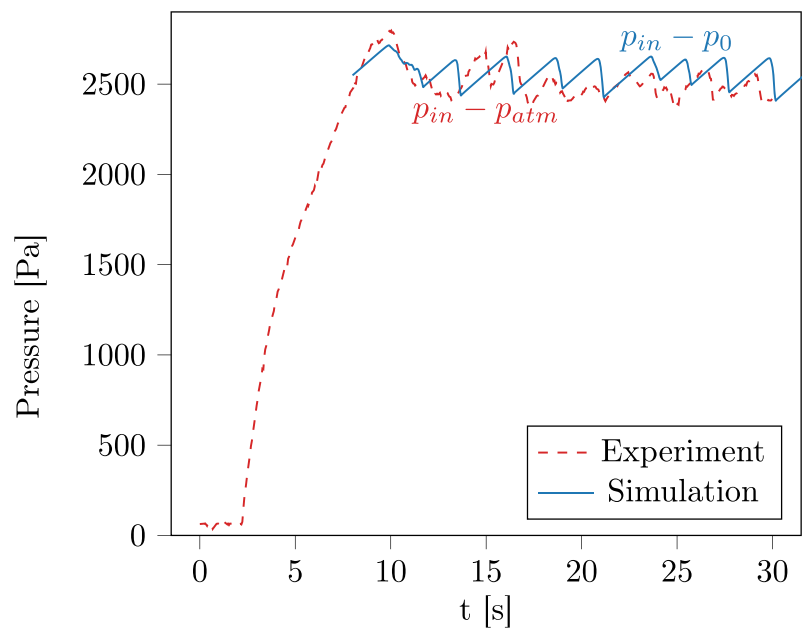

Fig. 12 Comparison of the differential pressure signals in the injection pipe obtained numerically and experimentally for the case $h_{\mathrm{g}}=4.5 \mathrm{~cm}$ and $I=13 \mathrm{~mL} / \mathrm{min}\left(\mathrm{Ca}=1.2 \times 10^{-2}, \mathrm{Re}_{s}=196.3\right.$ and $\Delta t=$ $\left.1.125 \times 10^{-4} \mathrm{~s}\right) . p_{0}$ is the reference pressure for the simulation, and $p_{\text {atm }}$ is the atmospheric pressure

As it has already been observed [62], the fluidized area starts at the top of the bed. Figure 14 shows the perturbation of the initial arrangement after the passage of one, two and three bubbles. It can be seen that bubbles carry grains along the channels they create. It forces the grains near the channels to slide downward to fill the void, while the grains brought in the wake of the bubble settle at the top of the bed. This forms a mixed pack of grains that grows from above downwards. The sliding areas are also visible through the curvature of the different layers.

\subsubsection{Stress tensor inside the granular bed}

Achieving simulations is also the opportunity to give insight into the stress field inside the granular bed. Let us remind that we consider grains as rigid bodies and compute the contact reactions using NSCD method. The knowledge of the contact reactions between the grains enables to estimate the areas submitted to strong stresses. It is possible to represent the contact network into the granular medium; however, due to the grains size it should be more clever to make use of a continuous representation of the constraint. Let us consider the granular medium as a continuous medium. It is then possible to define some points on a regular grid and to eval- 


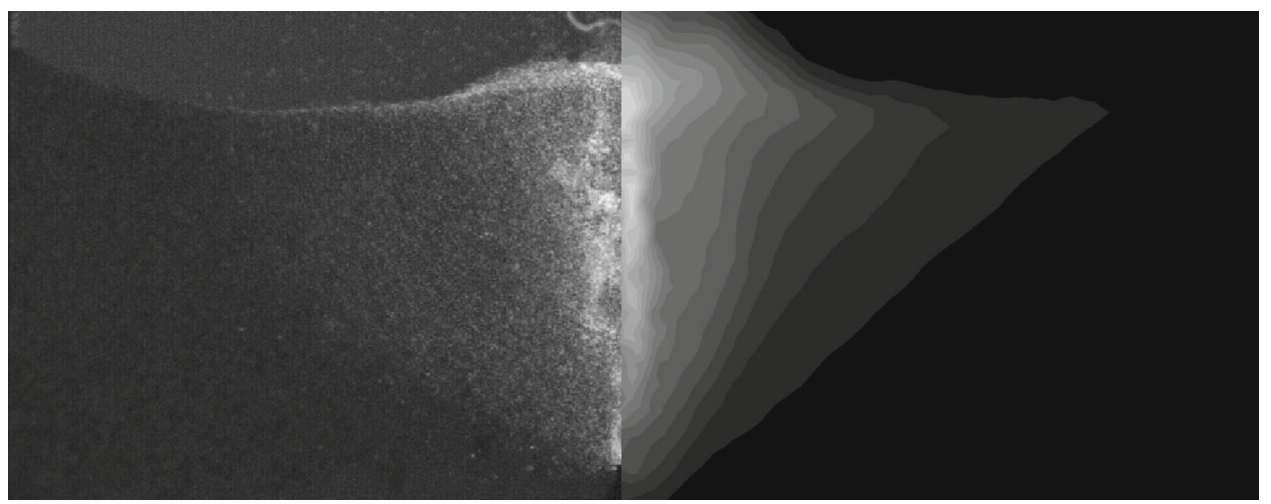

Fig. 13 Representation of the area where the motion are dominant after $30.5 \mathrm{~s}$ for the case $h_{\mathrm{g}}=4.5 \mathrm{~cm}$ and $I=6.5 \mathrm{~mL} / \mathrm{min}$. The experiment (left) is compared to the simulation (right). In the simulation, the Eulerian representation of the grains velocity is cumulated over time to

uate the stress tensor in a reference volume $\Omega$ around these points by summing all the contact efforts in this volume [9]. In the case of spherical grains, the stress tensor is given by the external product between the reaction force $\boldsymbol{R}^{c}$ and the centre-to-centre vector $\boldsymbol{l}^{c}$ of each contact $c$ inside the reference volume:

$S_{i j}=\frac{1}{\Omega} \sum_{c \in \Omega} R_{i}^{c} l_{j}^{c}$,

where the $R_{i}^{c}$ and $R_{j}^{c}$ are used to indicate the components of the vectors in the $i$ and $j$ directions, respectively.

Figure 15 shows the stress tensor at different times of the simulation. Snapshots at time 17.6 s, 18.6 s and 19.8 s show the three main steps of the first bubble ascent. The pressure increases in the pipe so that the air is pushed inside the grains. The cavity creation generates stresses around it due to the rearrangement of the granular matrix. Then, the bubble ascents in the granular bed. The grains on both sides of the bubble slowly slide downwards to fill the gap. There are fewer contacts in this fluidized area during the deposition, and as a result, the main stresses are located above the air cavity. The air finally escapes and the suspended grains settle in the cavity area. The main stresses are now located along the sliding planes. After the passage of the first bubble, the main stresses are due to the weight of the grains and located in the compact areas of the bed. Snapshot at time 22.6 s shows the typical stress state during the passage of the following bubbles. We observe stresses that grow linearly from the top to the bottom in the compact areas of the bed with some variations near the injector. At this point, the stresses tend to increase at the formation of a cavity but the dynamics observed for the first bubble does not repeat itself. The formation of the fluidized area reduces the propagation of the stresses in the granular bed for the following bubbles. determine areas where the motion is dominant, while in the experiment we make use of the cumulative pixels intensity difference between two successive image captures

\subsubsection{Inflow rate effect}

The inflow rate impacts the fluidized area and the invasion process. Comparisons between experiments and simulations achieved in this last part stage an immersed granular bed of height $h_{\mathrm{g}}=9 \mathrm{~cm}$ with different inflow rates (Fig. 16). The initial bed consists of 40,777 grains with a diameter uniformly distributed in the range $500<d_{s}<600 \mu \mathrm{m}$. Increasing the inflow rate clearly increases the bubble frequency as we already observed for the case with $h_{\mathrm{g}}=4.5$ $\mathrm{cm}$. Figure 16 shows the evolution of each system during $1 \mathrm{~s}$. It can be seen that experiments and simulations give similar results and bubble frequencies that are quite the same for the different inflow rates even if it should be pointed out that the frequency tends to be a little bit smaller in the simulations.

The air invasion creates a fluidized area in the granular bed as we explained above. This fluidized area has an impact on the motion of the grains and the stresses. Changing the inflow rate, i.e. the bubble frequency, has an impact on the fluidized area and the grains motion. It has been shown that after a long time, the fluidized area reaches a steady state [62], so that it is possible to characterize each system by the morphology of this steady state area. One feature of interest is the shape of the fluid-grains interface. During the air invasion process, the air flow creates a bump at the middle of the fluid-grains interface, while the borders shape depends on the sliding and deposition rates of the grains. Due to the computational time, we only focus on the first seconds of the invading process so that we do not reach a steady state for the fluidized area but it is still possible to compare the interface shapes obtained numerically and experimentally during the transition phase at the same time (Fig. 17).

At low inflow rate, the interface takes a $W$-like shape. The middle is rounded due to the volume of air inside the granular bed while the borders are slightly inclined. By increasing 

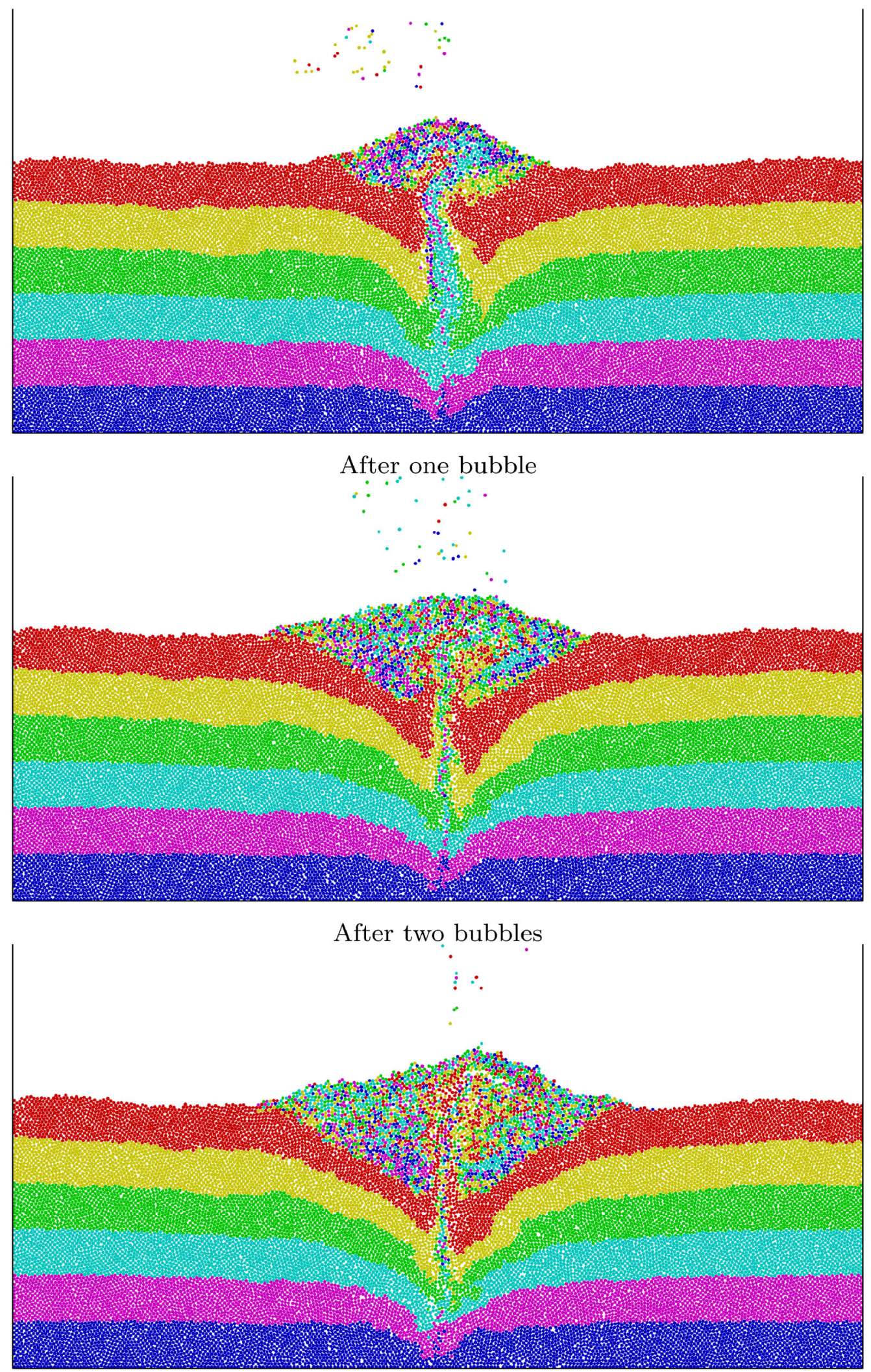

After three bubbles

Fig. 14 Configuration of the grains inside the bed after the passage of one, two and three bubbles for the case $h_{\mathrm{g}}=4.5 \mathrm{~cm}$ and $I=6.5 \mathrm{~mL} / \mathrm{min}$. Grains are coloured with respect to their initial height 
Fig. 15 Representation of the stress tensor inside the granular medium at different times. The stress tensor is represented by ellipses giving the eigenvectors direction and coloured with respect to their greatest eigenvalue $\lambda_{\max }$. (Color figure online)
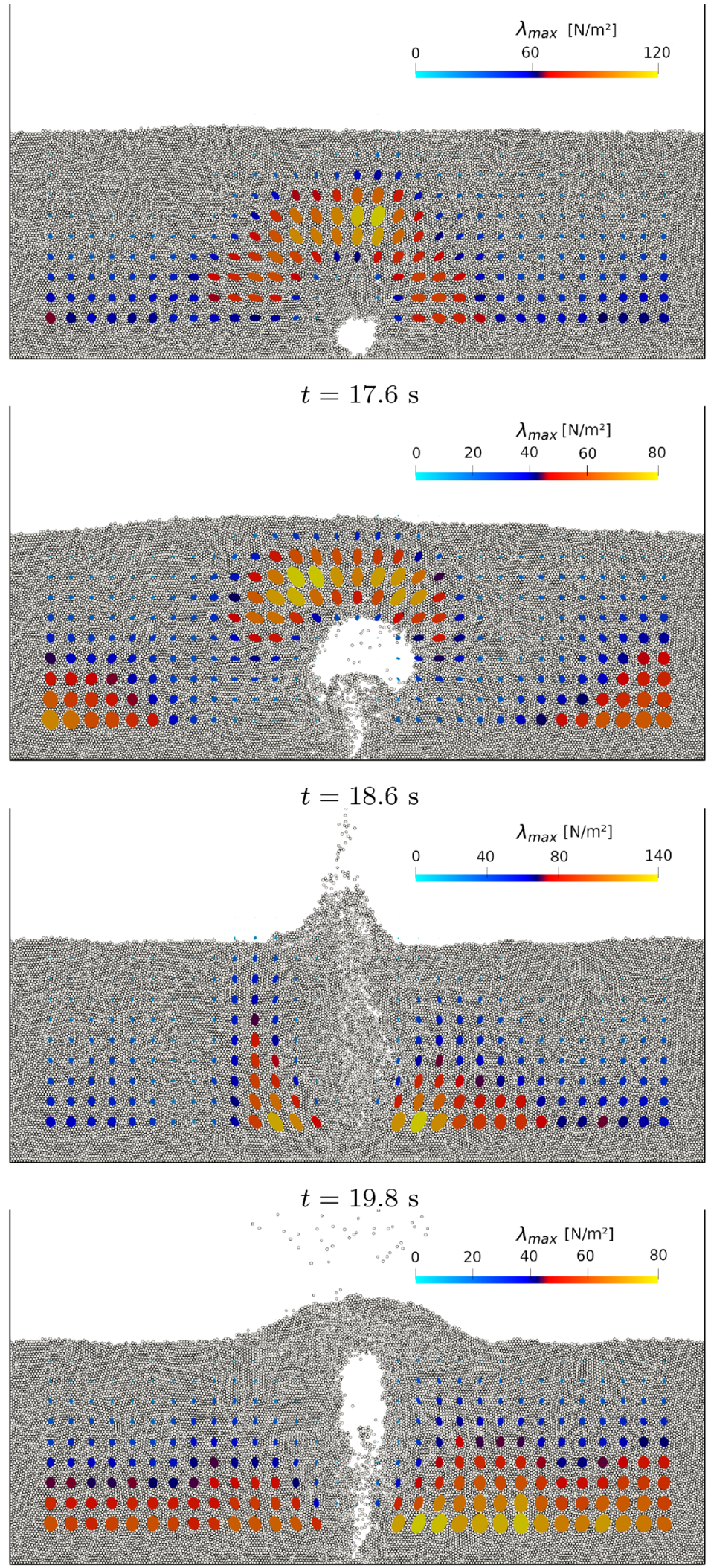

$t=22.6 \mathrm{~s}$ 
$I=50 \mathrm{~mL} / \mathrm{min}$

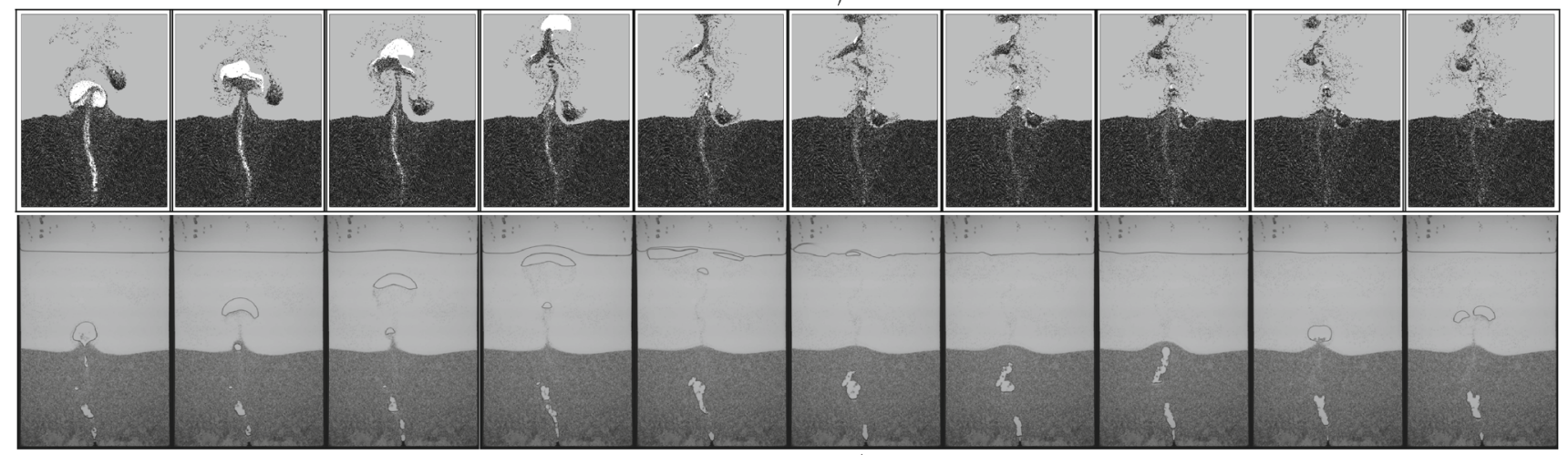

\section{$I=100 \mathrm{~mL} / \mathrm{min}$}
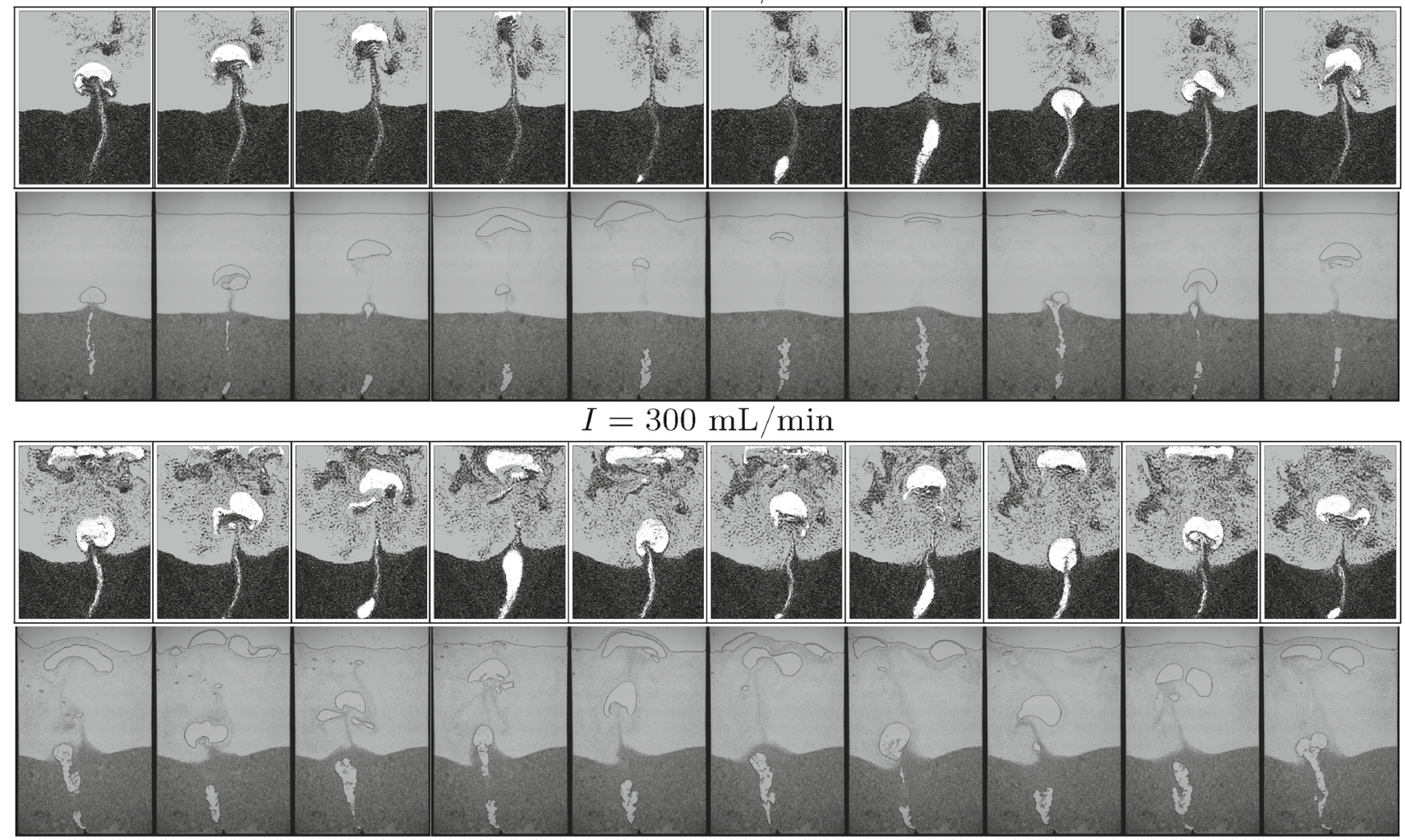

Fig. 16 Comparison between simulations and experiments of the air invading a granular bed of height $h_{\mathrm{g}}=9 \mathrm{~cm}$ at inflow rate $I=$ $50 \mathrm{~mL} / \mathrm{min}$ (top), $I=100 \mathrm{~mL} / \mathrm{min}$ (middle) and $I=300 \mathrm{~mL} / \mathrm{min}$

the inflow rate, the interface shape is sharper. The sliding of grains from the borders to the fluidized area is increased due to the intensity of the air flow. Using an even larger inflow rate, a great number of grains are blown by the air flow and suspended in the fluid. As a result, the sliding process is more important and the interface has a $V$-like shape.

This evolution of the interface shape is the same in the experiments and in the simulations. However, it seems that the number of suspending grains is higher in the simulations. This could be attributed to the solid volume fraction in the (bottom). For each case, the simulation corresponds to the first row of images. The time step between two successive images is $0.1 \mathrm{~s}$

granular bed. In two dimensions, it is possible to reach more compact piles than in three dimensions. A higher pressure is required before the air is pushed inside the bed. It creates bigger bubbles that are able to carry more grains in their wake. This also could be an additional explanation for the smaller frequency of bubble emissions in the simulations. The higher pressure peak we observe for the first bubble emission in the simulations is a proof of the impact of the solid volume fraction on the air invasion process. The pile is 
Fig. 17 Granular bed states for $h_{\mathrm{g}}=9 \mathrm{~cm}$ and $I=50 \mathrm{~mL} / \mathrm{min}$ $\left(\mathrm{Ca}=1.4 \times 10^{-2}, \mathrm{Re}_{s}=229.0\right.$ and $\Delta t=2.5 \times 10^{-4} \mathrm{~s}$ ),

$I=100 \mathrm{~mL} / \mathrm{min}$

$\left(\mathrm{Ca}=2.4 \times 10^{-2}, \mathrm{Re}_{s}=392.5\right.$ and $\Delta t=1.125 \times 10^{-4} \mathrm{~s}$ ) and $I=300 \mathrm{~mL} / \mathrm{min}$

$\left(\mathrm{Ca}=2.6 \times 10^{-2}, \mathrm{Re}_{s}=425.2\right.$ and $\Delta t=5.625 \times 10^{-5} \mathrm{~s}$ ) after $10 \mathrm{~s}$. The fluid-grains interface takes a flat $W$-like shape for the smaller inflow rate, while for the intermediate inflow rate the angles are sharper. With the higher inflow rate, the number of suspended grains is more important so that the sliding planes ended around the air invasion channel forming a $V$-like shape interface

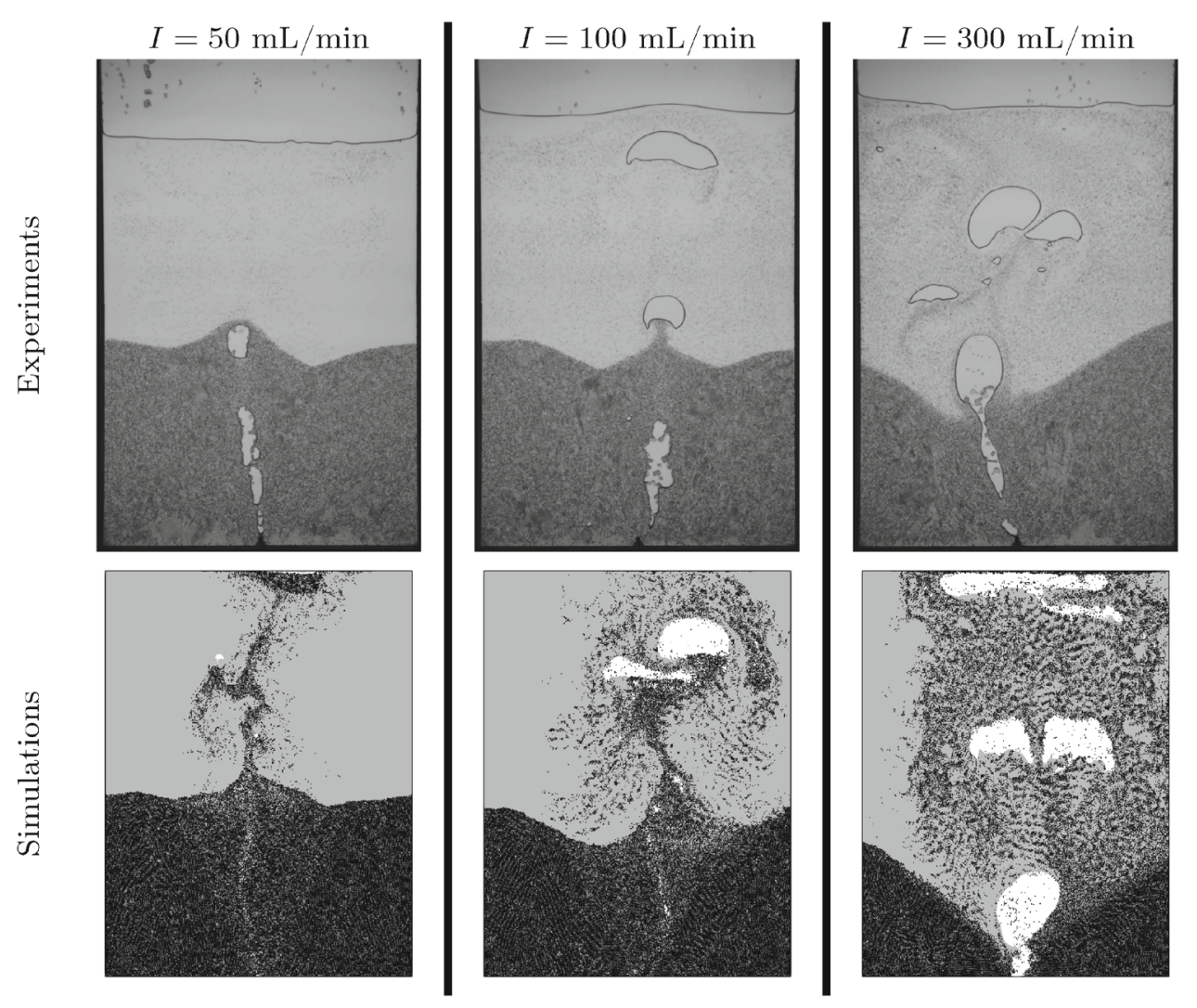

more compact at the beginning of the simulation than after the passage of the first bubble.

The two-dimensional nature of the simulations might be a problem for large pressure in the injection pipe. The loss of one dimension changes the flow dynamics by reducing the degrees of freedom. The validity of the two-dimensional simulations without additional parametrizations appears to be restricted to regimes close to the one the parameters have been calibrated for. At low inflow rate, this difference seems to be negligible so that the main features of the flow are well captured in the simulations.

The drag force could also be a source of error. It is computed based on an empirical formula that may be unsuitable for all the flow regimes encountered in the computed cases. Moreover, the drag coefficient (19) relates to the density and the viscosity that relate in turn to the phase indicator function. As discussed above, this leads to a drag formula that increases slightly near areas where the phase indicator function has a value around 0.5 . Due to the coarse resolution of the liquid-gas interface, the interface is diffusively defined and it is susceptible to cause an increase in the number of grains carried in the wake of the bubbles.

\section{Conclusion}

An implementation of an unresolved CFD-DEM method is described for the simulation of gas-liquid-solid flows without interface reconstruction. Grains are represented as discrete Lagrangian particles on which forces are applied. Deformations and overlapping of the grains are prevented by the non-smooth contact dynamics method. The fluid is computed through an Eulerian representation of the liquid-gas mixture. The distinction between gas and liquid phases is based on a continuous phase indicator function. Its time and spatial evolutions are dictated by the fluid bulk velocity. This phase indicator function is used to determine the density and viscosity fields. The surface tension force is applied at the gas-liquid interface. In the presence of grains, continuous fluid equations have to be weighted by the effective volume left for the fluid in the reference volume.

The finite element method with equal-order linear interpolation functions is used. This leads to a method, although fast and convenient, that requires the addition of stabilization terms to suppress spurious modes. Stabilization terms add some pressure diffusion to fill the rank deficiency of the problem matrix and some velocity diffusion in the flow direction for convection-dominated flows. These terms are based on equation residuals to obtain a consistent method in which the stabilization terms vanish with the solution convergence. 
The equations and numerical methods used in this work have been validated on simplified cases to prove their accuracy. This provides good results concerning the behaviour of the different strategies that have been implemented to face instability problems.

The model has been then applied to the more complex case of the air invading an immersed granular bed. Experiments have been carried out in a Hele-Shaw cell to identify the key parameters and processes of the system. This quasi-twodimensional device has been considered to ease comparisons with two-dimensional simulations and determine the features that were reproducible in two dimensions. It has particularly pointed out that the inflow boundary condition has a crucial influence on the flow dynamics inside the cell. We made use of the experiment carried out with the lowest inflow rate to calibrate the influencing parameters of the model before validating it on experiment carried out with larger inflow rates. In each case, we found that simulations were in good agreement with the experiments. The bubble morphologies and the invasion dynamics have been studied to assess the good concordance. The simulations have been used to give insight in the fluidized area formation and the stresses that are propagated in the granular matrix. Simulations show that the fluidized area grows from the top of the granular bed due to the higher mobility of the grains as it was already pointed out in previous studies. It also shows that the stresses are more important for the first bubble because the initial state of the grains is more compact. After the passage of the first bubble, the pack is more loose preventing the propagation of the constraints.

The shape of the fluid-grains interface has also been observed for different inflow rates. It has been shown that after some time this interface tends to a $W$-like shape due to the deposition and suspension cycles. Increasing the inflow rate sharpens the angles of the fluid-grains interface until there are so many suspended grains that the interface takes a $V$-like shape formed by the sliding planes around the channel created by the air.

However, little differences have been observed between the simulations and the experiments. The period of the pressure signal in the injection pipe tends to be longer in the simulations. This may be caused by the fluid representation. In a model based on a coarse fluid representation scale, it is quite obvious that only phenomena happening at a sufficiently large scale will be captured. It is then possible that the period of the pressure signal is longer because it is only based on the time separating two large-scale events. This problem could be overcome by the use of smaller elements in the areas of interest.

In our model, the size of the numerical cells must be greater than the grain size because of the averaging process of the equations that requires nonzero fluid volume fraction. It should be possible to develop a model based on a con- tinuous transition between a microscale representation and a mesoscale representation. For example, we could consider a different averaging scale so that the fluid volume fraction is computed at a large scale as a continuous nonzero field, while the averaged Navier-Stokes equations are discretized at a smaller scale.

Based on a kernel approximation similar to the one achieved in the smoothed particle hydrodynamics (SPH) [23] to evaluate field values at Lagrangian particles location, it is possible to achieve the volume averaging at a scale independent from the fluid discretization to obtain a semi-resolved CFD-DEM models [65]. Such a method has already been used to compute particle-laden flows [6] but faces the same issues than SPH methods [20].

This choice may lead to the loss of the consistency because the averaging volume used for the fluid volume fraction is truncated near the boundaries [8]. In two-dimensional simulations, the solid volume fraction can reach values up to 0.9 for hexagonal arrangements. As a result, in the preliminary tests we achieved in this way, we observed that the change in the solid volume fraction due to the truncation of the kernel support highly affects the air flow near the injector boundaries. Moreover, the use of a kernel function is not appropriate to the semi-implicit scheme developed to compute the drag force. It makes use of an implicit fluid velocity so that averaging it on a kernel support larger that the mesh size will result in a loss of the coefficient matrix sparsity and a high computational cost. The use of nested meshes in the way of the dual grid presented by [43] could solve the mentioned problems. On the one hand, the use of the coarse grid as averaging support prevents the inconsistency near the walls because the boundaries match the kernel supports. On the other hand, it gives a local pattern to preserve the coefficient matrix sparsity. The development of such a model should be investigated in the future. It should give us the opportunity to capture the small-scale events and to represent the boundary asperities that have dimensions equal to the grain size.

Another difference observed is the greater proportion of grains that are suspended by the ascent of a bubble observed in the simulations. This could be explained by the smaller solid volume fraction that can be reached in two dimensions. It results that the granular bed is more compact in the simulations than in the experiments increasing the constraint the gas has to overcome to invade the medium. The higher the pressure, the larger the bubbles. The air is then able to carry more grains in its wake. Simulations achieved with a correction on the fluid volume fraction or computations achieved in three dimensions should give even better agreements with the experiments. However, the computation time is rapidly becoming an issue. This study is limited to the start of the invasion process because of the computation time required to reach the steady-state of the fluidized area. This model has been proved able to reproduce the air invasion in an immersed 
granular bed and an improvement in the computation speed should give us the opportunity in the future to give more insights in the fluidized area growth process at short and long times.

Acknowledgements Matthieu Constant is a Research Fellow with the Belgium Fund for Research in Industry and Agriculture (FRIA).

Funding This study was funded by the F.R.S.-FNRS through a FRIA Grant (29627518).

Code availability The source code of the MigFlow software used to produce the results presented in this article is released under the GPL license and available here https://git.immc.ucl.ac.be/fluidparticles/migflow/-/ tags/amc2ThreePhases.

\section{Compliance with ethical standards}

Conflict of interest The authors declare that they have no conflict of interest.

\section{References}

1. Anderson T, Jackson R (1968) A fluid mechanical description of fluidized beds: stability of the uniform state of fluidization. I\&EC Fundam 7:12-21

2. Babuska I (1973) The finite element method with lagrangian multipliers. Numer Math 20(3):179-192

3. Brackbill JU, Kothe DB, Zemach C (1992) A continuum method for modeling surface tension. J Comput Phys 100(2):335-354

4. Brezzi F, Pitkaranta J (1984) On the stabilization of the finite element approximations of the Stokes equations. In: Hackbush W (ed) Efficient solution of elliptic systems, vol 10. Vieweg, Braunschweig, pp 11-19

5. Brooks AN, Hughes TJ (1982) Streamline upwind/PetrovGalerkin formulations for convection dominated flows with particular emphasis on the incompressible Navier-Stokes equations. Comput Methods Appl Mech Eng 32(1-3):199-259

6. Capecelatro J, Desjardins O (2013) An Euler-Lagrange strategy for simulating particle-laden flows. J Comput Phys 238:1-31

7. Casagrande MV, Alves JL, Silva CE, Alves FT, Elias RN, Coutinho AL (2017) A hybrid FEM-DEM approach to the simulation of fluid flow laden with many particles. Comput Part Mech 4(2):213-227

8. Chen J, Beraun J, Carney T (1999) A corrective smoothed particle method for boundary value problems in heat conduction. Int $\mathrm{J}$ Numer Methods Eng 46(2):231-252

9. Chetouane B, Dubois F, Vinches M, Bohatier C (2005) NSCD discrete element method for modelling masonry structures. Int $\mathrm{J}$ Numer Methods Eng 64(1):65-94

10. Chu K, Wang B, Yu A, Vince A (2009) CFD-DEM modelling of multiphase flow in dense medium cyclones. Powder Technol 193(3):235-247. https://doi.org/10.1016/j.powtec.2009. 03.015 (Special Issue: Discrete element methods: the 4th international conference on discrete element methods, Brisbane, August 2007)

11. Chu K, Yu A (2008) Numerical simulation of complex particle-fluid flows. Powder Technol 179(3):104-114. https://doi.org/10.1016/j. powtec.2007.06.017 (WCPT5 papers presented at the 5th world conference of particle technology (WCPT5), Orlando, Florida, April 23-27 2006 5th world conference of particle technology (WCPT5))
12. Constant M, Dubois F, Lambrechts J, Legat V (2018) Implementation of an unresolved stabilised FEM-DEM model to solve immersed granular flows. Comput Part Mech. https://doi.org/10. 1007/s40571-018-0209-4

13. Crowe CT, Schwarzkopf JD, Sommerfeld M, Tsuji Y (2011) Multiphase flows with droplets and particles. CRC Press, Berlin

14. Cundall PA, Strack ODL (1979) A discrete numerical model for granular assemblies. GAl'otechnique 29(1):47-65

15. DallaValle JM, Klemin A (1943) Micromeritics: the technology of the particles. Pitman Publishing Corporation, Berlin

16. Darcy HPG (1856) Les Fontaines Publiques de la Villes de Dijon. Victor Dalmont, Paris

17. Deen N, Annaland MVS, de Hoef MV, Kuipers J (2007) Review of discrete particle modeling of fluidized beds. Chem Eng Sci 62:28 44

18. Di Felice R, Rotondi M (2012) Fluid-particle drag force in binarysolid suspensions. Int J Chem React Eng. https://doi.org/10.1515/ $1542-6580.3000$

19. Epstein N, Grace JR (2010) Spouted and spout-fluid beds: fundamentals and applications. Cambridge University Press, Cambridge

20. Esteghamatian A, Euzenat F, Hammouti A, Lance M, Wachs A (2018) A stochastic formulation for the drag force based on multiscale numerical simulation of fluidized beds. Int J Multiph Flow 99:363-382

21. Forchheimer P (1901) Wasserbewegung durch Boden, 45 edition edn. Zeitschrift des Vereines Deutscher Ingenieuer

22. Gidaspow D (1994) Multiphase flow and fluidization: continuum and kinetic theory descriptions. Academic Press, Berlin

23. Gingold RA, Monaghan JJ (1977) Smoothed particle hydrodynamics: theory and application to non-spherical stars. Mon Not R Astron Soc 181(3):375-389

24. Grosshans H, Movaghar A, Cao L, Oevermann M, SzAạsz RZ, Fuchs L (2016) Sensitivity of VOF simulations of the liquid jet breakup to physical and numerical parameters. Comput Fluids 136:312-323. https://doi.org/10.1016/ j.compfluid.2016.06.018. http://www.sciencedirect.com/science/ article/pii/S0045793016302031

25. Happel J (1958) Viscous flow in multiparticle systems: slow motion of fluids relative to beds of spherical particles. AIChE J 4(2):197201

26. Hirt C, Nichols B (1981) Volume of fluid (VOF) method for the dynamics of free boundaries. J Comput Phys 39(1):201-225. https://doi.org/10.1016/0021-9991(81)90145-5

27. $\mathrm{Hu} \mathrm{HH}$ (1996) Direct simulation of flows of solid-liquid mixture. Int J Multiph Flow 22(2):335-352

28. Hughes TJ, Franca LP, Balestra M (1986) A new finite element formulation for computational fluid dynamics: V. circumventing the BabuÅąka-Brezzi condition: a stable Petrov-Galerkin formulation of the Stokes problem accommodating equal-order interpolations. Comput Methods Appl Mech Eng 59(1):85-99. https://doi.org/10. 1016/0045-7825(86)90025-3

29. Jamet D, Torres D, Brackbill J (2002) On the theory and computation of surface tension: the elimination of parasitic currents through energy conservation in the second-gradient method. J Comput Phys 182(1):262-276

30. Jean M (1999) The non-smooth contact dynamics method. Comput Methods Appl Mech Eng 177(3-4):235-257

31. Jing L, Kwok C, Leung Y, Sobral Y (2016) Extended CFD-DEM for free-surface flow with multi-size granules. Int J Numer Anal Meth Geomech 40(1):62-79

32. Kafui K, Thornton C, Adams M (2002) Discrete particlecontinuum fluid modelling of gas-solid fluidised beds. Chem Eng Sci 57(13):2395-2410

33. Kong XZ, Kinzelbach W, Stauffer F (2010) Morphodynamics during air injection into water-saturated movable spherical granulates. Chem Eng Sci 65(16):4652-4660. https://doi.org/10.1016/ 
j.ces.2010.05.007. http://www.sciencedirect.com/science/article/ pii/S0009250910002976

34. KAûhl M, Lu G, Third J, Pruessmann KP, Müller C, (2014) Magnetic resonance imaging (MRI) of jet height hysteresis in packed beds. Chem Eng Sci 109:276-283. https://doi.org/10.1016/ j.ces.2014.01.038. http://www.sciencedirect.com/science/article/ pii/S0009250914000517

35. Lafaurie B, Nardone C, Scardovelli R, Zaleski S, Zanetti G (1994) Modelling merging and fragmentation in multiphase flows with surfer. J Comput Phys 113(1):134-147

36. Le Beau GJ, Tezduyar TE (1991) Finite element computation of compressible flows with the SUPG formulation. In: Dhaubhadel MN, Engelman MS, Reddy JN (eds) Advances in finite element analysis in fluid dynamics, FED, vol 123. ASME, New York, 1991, pp 21-27

37. Li J, Kuipers JAM (2003) Gas-particle interactions in dense gasfluidized beds. Chem Eng Sci 58(3-6):711-718

38. McNamara S, Young WR (1992) Inelastic collapse and clumping in a one-dimensional granular medium. Phys Fluids A 4(3):496-504

39. Park KM, Yoon HS, Kim MI (2018) CFD-DEM based numerical simulation of liquid-gas-particle mixture flow in dam break. Commun Nonlinear Sci Numer Simul 59:105-121

40. Peng Z, Doroodchi E, Luo C, Moghtaderi B (2014) Influence of void fraction calculation on fidelity of CFD-DEM simulation of gas-solid bubbling fluidized beds. AIChE J 60(6):2000-2018

41. Peskin CS (2002) The immersed boundary method. Acta Numer 11:479-517

42. Popinet S, Zaleski S (1999) A front-tracking algorithm for accurate representation of surface tension. Int J Numer Methods Fluids 30(6):775-793

43. Pozzetti G, Peters B (2018) A multiscale DEM-VOF method for the simulation of three-phase flows. Int J Multiph Flow 99:186-204

44. Prosperetti A (2002) Navier-Stokes numerical algorithms for freesurface flow computations: an overview. In: Rein M (eds) Dropsurface interactions. CISM International Centre for Mechanical Sciences (Courses and Lectures), vol 456. Springer, Vienna. https:// doi.org/10.1007/978-3-7091-2594-6_8

45. Richardson J, Zaki W (1954) The sedimentation of a suspension of uniform spheres under conditions of viscous flow. Chem Eng Sci 3(2):65-73. https://doi.org/10.1016/00092509(54)85015-9 http://www.sciencedirect.com/science/article/ pii/0009250954850159

46. Rudman M (1998) A volume-tracking method for incompressible multifluid flows with large density variations. Int J Numer Methods Fluids. https://doi.org/10.1002/(sici)10970363(19980815)28:2<357::aid-fld750>3.0.co;2-d

47. Sangani AS, Acrivos A (1982) Slow flow past periodic arrays of cylinders with application to heat transfer. Int J Multiph Flow 8(3):193-206

48. Semer R, Adams J, Reddy K (1998) Technical note an experimental investigation of air flow patterns in saturated soils during air sparging. Geotech Geol Eng 16(1):59-75. https://doi.org/10.1023/ A: 1008884213834

49. Shakib F (1989) Finite element analysis of the compressible Euler and Navier-Stokes equations. Stanford University, Stanford, California

50. Sun X, Sakai M (2015) Three-dimensional simulation of gassolid-liquid flows using the DEM-VOF method. Chem Eng Sci 134:531-548

51. Svensen H, Jamtveit B, Planke S, Chevallier L (2006) Structure and evolution of hydrothermal vent complexes in the karoo basin, south africa. J Geol Soc 163(4):671-682

52. Taghipour F, Ellis N, Wong C (2005) Experimental and computational study of gas-solid fluidized bed hydrodynamics. Chem Eng Sci 60(24):6857-6867
53. Tamayol A, Bahrami M (2009) Analytical determination of viscous permeability of fibrous porous media. Int J Heat Mass Transf 52(910):2407-2414

54. Tamayol A, Bahrami M (2011) Transverse permeability of fibrous porous media. Phys Rev E 83(4):046314

55. Terfous A, Hazzab A, Ghenaim A (2013) Predicting the drag coefficient and settling velocity of spherical particles. Powder Technol 239:12-20

56. Tezduyar T, Sathe S (2003) Stabilization parameters in SUPG and PSPG formulations. J Comput Appl Mech 4(1):71-88

57. Tezduyar TE, Mittal S, Ray S, Shih R (1992) Incompressible flow computations with stabilized bilinear and linear equal-orderinterpolation velocity-pressure elements. Comput Methods Appl Mech Eng 95(2):221-242

58. Tezduyar TE, Osawa Y (2000) Finite element stabilization parameters computed from element matrices and vectors. Comput Methods Appl Mech Eng 190(3-4):411-430

59. Tsuji Y, Kawaguchi T, Tanaka T (1993) Discrete particle simulation of two dimensional fluidized bed. Powder Technol 77(1):79-87

60. van der Hoef M, van Sint Annaland M, Ye M, Andrews A, Sundaresan S, Kuipers J (2006) Multiscale modeling of gas-fluidized beds. Adv Chem Eng 31:65-149. https://doi.org/10.1016/S00652377(06)31002-2

61. Vångö M, Pirker S, Lichtenegger T (2018) Unresolved CFD-DEM modeling of multiphase flow in densely packed particle beds. Appl Math Model 56:501-516

62. Varas G, Géminard JC, Vidal V (2013) Air invasion in a granular layer immersed in a fluid: morphology and dynamics. Granular Matter 15(6):801-810. https://doi.org/10.1007/s10035-013-04357

63. Varas G, Vidal V, Géminard JC (2011) Morphology of air invasion in an immersed granular layer. Phys Rev E 83:061302. https://doi. org/10.1103/PhysRevE.83.061302

64. van Wachem B, Almstedt A (2003) Methods for multiphase computational fluid dynamics. Chem Eng J 96(1-3):81-98. https://doi. org/10.1016/j.cej.2003.08.025 (Festschrift Prof. Cor M. van den Bleek)

65. Wang Z, Teng Y, Liu M (2019) A semi-resolved CFD-DEM approach for particulate flows with kernel based approximation and Hilbert curve based searching strategy. J Comput Phys 384:151169

66. Wen CY, Yu HY (1966) Chemical engineering progress symposium series. Mech Fluid 62:100-111

67. Whitaker S (1986) Flow in porous media I: a theoretical derivation of Darcy's law. Transp Porous Media 1(1):3-25

68. Xu BH, Yu AB (1997) Numerical simulation of the gas-solid flow in a fluidized bed by combining discrete particle method with computational fluid dynamics. Chem Eng Sci 52(16):2785-2809

69. Zhang J, Fan LS, Zhu C, Pfeffer R, Qi D (1999) Dynamic behaviour of collision of elastic spheres in viscous fluids. Powder Technol 106(1-2):98-109

70. Zhong W, Yu A, Zhou G, Xie J, Zhang H (2016) CFD simulation of dense particulate reaction system: approaches, recent advances and applications. Chem Eng Sci 140:16-43. https://doi.org/10.1016/j. ces.2015.09.035

71. Zhu HP, Zhou ZY, Yang RY, Yu AB (2007) Discrete particle simulation of particulate system: theoretical developments. Chem Eng Sci 62(13):3378-3396

72. Zhu HP, Zhou ZY, Yang RY, Yu AB (2008) Discrete particle simulation of particulate system: a review of major applications and finings. Chem Eng Sci 63:5728-5770 